(c) 2004 International Press

Adv. Theor. Math. Phys. 8 (2004) 735-777

\title{
The notion of observable in the
}

\section{covariant Hamiltonian formalism}

\section{for the calculus of variations with}

\author{
several variables
}

\author{
Frédéric Hélein \\ Institut de Mathématiques de Jussieu, UMR 7586, \\ Université Denis Diderot-Paris 7, Site de Chevaleret, \\ 16 rue Clisson 75013 Paris, France \\ helein@math.jussieu.fr \\ Joseph Kouneiher \\ LUTH, CNRS UMR 8102, Observatoire de Paris - section Meudon, \\ 5 Place Jules Janssen, 92195 Meudon Cedex, France \\ kouneiher@paris7.jussieu.fr
}

\begin{abstract}
This papers is concerned with multisymplectic formalisms which are the frameworks for Hamiltonian theories for fields theory. Our main purpose is to study the observable $(n-1)$-forms which allows one to construct observable functionals on the set of solutions of the Hamilton equations by integration. We develop here two different points of view: generalizing the law $\{p, q\}=1$ or the law $d F / d t=\{H, F\}$. This leads to two possible definitions; we explore the relationships and the differences between these two concepts. We show that - in contrast with the de Donder-Weyl theory - the two definitions coincides in the Lepage-Dedecker theory.
\end{abstract}

e-print archive: http://lanl.arXiv.org/abs/math-phys/0401047 


\section{Introduction}

Multisymplectic formalisms are the frameworks for finite dimensional formulations of variational problems with several variables (or field theories for physicists)analogous to the well-known Hamiltonian theory of point mechanics. They are based on the following analogues of symplectic forms: given a differential manifold $\mathcal{M}$ and $n \in \mathbb{N}$ a smooth $(n+1)$-form $\Omega$ on $\mathcal{M}$ is a multisymplectic form if and only if $\Omega$ is non degenerate (i.e., $\forall m \in \mathcal{M}$, $\forall \xi \in T_{m} \mathcal{M}$, if $\left.\xi\right\lrcorner \Omega_{m}=0$, then $\left.\xi=0\right)$ and closed. We call $(\mathcal{M}, \Omega)$ a multisymplectic manifold. Then one can associates to a Lagrangian variational problem a multisymplectic manifold $(\mathcal{M}, \Omega)$ and a Hamiltonian function $\mathcal{H}$ on $\mathcal{M}$ s.t. any solution of the variational problem is represented by a solution of a system of generalized Hamilton equations. Geometrically this solution is pictured by an $n$-dimensional submanifold $\Gamma \subset \mathcal{M}$ s.t. $\forall m \in \mathcal{M}$ there exists a $n$-multivector $X$ tangent to $\mathcal{M}$ at $m$ s.t. $X\lrcorner \Omega=(-1)^{n} d \mathcal{H}$. We then call $\Gamma$ a Hamiltonian $n$-curve.

For example a variational problem on maps $u: \mathbb{R}^{n} \longrightarrow \mathbb{R}$ can written as the system $^{1}$

$$
\frac{\partial u}{\partial x^{\mu}}=\frac{\partial H}{\partial p^{\mu}}(x, u, p) \quad \text { and } \quad \sum_{\mu} \frac{\partial p^{\mu}}{\partial x^{\mu}}=-\frac{\partial H}{\partial u}(x, u, p) .
$$

The corresponding multisymplectic form is $\Omega:=d \theta$, where $\theta:=e \omega+p^{\mu} d u \wedge$ $\omega_{\mu}\left(\right.$ and $\omega:=d x^{1} \wedge \cdots \wedge d x^{n}$ and $\left.\left.\omega_{\mu}:=\partial_{\mu}\right\lrcorner \omega\right)$. The simplest example of such a theory was proposed by T. de Donder [5] and H. Weyl [30]. But it is a particular case of a huge variety of multisymplectic theories which were discovered by T. Lepage and can be described using a universal framework built by P. Dedecker [6], [14], [16].

The present paper, which is a continuation of [14] and [16], is devoted to the study of observable functionals defined on the set of all Hamiltonian $n$-curves $\Gamma$. An important class of such functionals can be constructed by choosing appropriate $(n-1)$-forms $F$ on the multisymplectic manifold $\mathcal{M}$ and a hypersurface $\Sigma$ of $\mathcal{M}$ which crosses transversally all Hamiltonian $n$ curves (we shall call slices such hypersurfaces). Then $\int_{\Sigma} F: \Gamma \longmapsto \int_{\Sigma \cap \Gamma} F$ is such a functional. One should however check that such functionals measure physically relevant quantities. The philosophy adopted here is inspired from quantum Physics: the formalism should provide us with rules for predicting

\footnotetext{
${ }^{1}$ An obvious difference with mechanics is that there is a dissymmetry between the "position" variable $u$ and the "momentum" variables $p^{\mu}$. Since (1) involves a divergence of $p^{\mu}$ one can anticipate that, when formulated in more geometrical terms, $p^{\mu}$ will be interpreted as the components of a $(n-1)$-form, whereas $u$ as a scalar function.
} 
the dynamical evolution of an observable. There are two ways to translate this requirement mathematically: first the "infinitesimal evolution" $d F(X)$ of $F$ along a $n$-multivector $X$ tangent to a Hamiltonian $n$-curve should be completely determined by the value of $d \mathcal{H}$ at the point - this leads to the definition of what we call an observable $(n-1)$-form (OF), the subject of Section 3; alternatively, inspired by an analogy with classical particle mechanics, one can assume that there exists a tangent vector field $\xi_{F}$ such that $\left.\xi_{F}\right\lrcorner \Omega+d F=0$ everywhere — we call such forms algebraic observable $(n-1)$-forms (AOF). This point of view will be investigated in Section 4 . We believe that the notion of AOF was introduced by W. Tulczyjew in 1968 [28] (see also [9], [11], [23]). To our knowledge the notion of OF was never considered before; it seems to us however that it is a more natural definition. It is easy to check that all AOF are actually OF but the converse is in general not true (see Section 4), as in particular in the de Donder-Weyl theory.

It is worth here insisting on the difference of points of view between choosing OF's or AOF's. The definition of OF is in fact the right notion if we are motivated by the interplay between the dynamics and observable functionals. It allows us to define a pseudobracket $\{\mathcal{H}, F\}$ between the Hamiltonian function and an $\mathrm{OF} F$ which leads to a generalization of the famous equation $\frac{d A}{d t}=\{H, A\}$ of the Hamiltonian mechanics. This is the relation

$$
d F_{\mid \Gamma}=\{\mathcal{H}, F\} \omega_{\mid \Gamma},
$$

where $\Gamma$ is a Hamiltonian $n$-curve and $\omega$ is a given volume $n$-form on spacetime (see Proposition 3.1). In contrast the definition of AOF's is the right notion if we are motivated in defining an analogue of the Poisson bracket between observable $(n-1)$-forms. This Poisson bracket, for two AOL $F$ and $G$ is given by $\left.\{F, G\}:=\xi_{F} \wedge \xi_{G}\right\lrcorner \Omega$, a definition reminiscent from classical mechanics. This allows us to construct a Poisson bracket on functionals by the rule $\left\{\int_{\Sigma} F, \int_{\Sigma} G\right\}: \Gamma \longmapsto \int_{\Sigma \cap \Gamma}\{F, G\}$ (see Section 4 ).

Note that it is possible to generalize the notion of observable $(p-1)$ forms to the case where $0 \leq p<n$, as pointed out recently in [21], [22]. For example the dissymmetry between variables $u$ and $p^{\mu}$ in system (1) suggests that, if the $p^{\mu}$ 's are actually the components of the observable $(n-1)$-form $p^{\mu} \omega_{\mu}, u$ should be an observable function. Another interesting example is the Maxwell action, where the gauge potential 1-form $A_{\mu} d x^{\mu}$ and the Faraday $(n-2)$-form $\star d A=\eta^{\mu \lambda} \eta^{\nu \sigma}\left(\partial_{\mu} A_{\nu}-\partial_{\nu} A_{\mu}\right) \omega_{\lambda \sigma}$ are also "observable", as proposed in [21]. Note that again two kinds of approaches for defining such observable forms are possible, as in the preceding paragraph: either our starting point is to ensure consistency with the dynamics (this leads us in Section 3 to the definition of OF's) or we privilege the definition which seems to be the more appropriate for having a notion of Poisson bracket 
(this leads us in Section 4 to the definition of AOF's). If we were to follow the second point of view we would be led to the following definition, in [21]: a $(p-1)$-form $F$ would be observable ("Hamiltonian" in [21]) if and only if there exists a $(n-p+1)$-multivector $X_{F}$ such that $\left.d F=(-1)^{n-p+1} X_{F}\right\lrcorner \Omega$. This definition has the advantage that - thanks to a consistent definition of Lie derivatives of forms with respect to multivectors due to W.M. Tulczyjew [29] - a beautiful notion of graded Poisson bracket between such forms can be defined, in an intrinsic way (see also [26], [7]). These notions were used successfully by [4] in treating the gauge theory and specifically the Yang-Mills case, and more generally by S. Hrabak for constructing a multisymplectic version of the Marsden-Weinstein symplectic reduction [19] and of the BRST operator [20]. Unfortunately such a definition of observable $(p-1)$-form would not have nice dynamical properties. For instance if $\mathcal{M}:=\Lambda^{n} T^{\star}\left(\mathbb{R}^{n} \times\right.$ $\mathbb{R})$ with $\Omega=d e \wedge \omega+d p^{\mu} \wedge d \phi \wedge \omega_{\mu}$, then the 0 -form $p^{1}$ would be observable, since $\left.d p^{1}=(-1)^{n} \frac{\partial}{\partial \phi} \wedge \frac{\partial}{\partial x^{2}} \wedge \cdots \wedge \frac{\partial}{\partial x^{n}}\right\lrcorner \Omega$, but there would be no chance for finding a law for the infinitesimal change of $p^{1}$ along a curve inside a Hamiltonian $n$-curve. By that we mean that there would be no hope for having an analogue of the relation (2) (Corollary 3.2).

That is why we have tried to base ourself on the first point of view and to choose a definition of observable $(p-1)$-forms in order to guarantee good dynamical properties, i.e., in the purpose of generalizing relation (2). A first attempt was in [14] for variational problems concerning maps between manifolds. We propose here another definition working for all Lepagean theories, i.e., more general. Our new definition works "collectively", requiring to the set of observable $(p-1)$-forms for $0 \leq p<n$ that their differentials form a sub bundle stable by exterior multiplication and containing differentials of observable $(n-1)$-forms (copolarization, Section 3). This definition actually merged out as the right notion from our efforts to generalize the dynamical relation (2). This is the content of Theorem 3.1.

Once this is done we are left with the question of defining the bracket between an observable $(p-1)$-form $F$ and an observable $(q-1)$-form $G$. We propose here a (partial) answer. In Section 4 we find necessary conditions on such a bracket in order to be consistent with the standard bracket used by physicists in quantum field theory. Recall that this standard bracket is built through an infinite dimensional Hamiltonian description of fields theory. This allows us to characterize what should be our correct bracket in two cases: either $p$ or $q$ is equal to $n$, or $p, q \neq n$ and $p+q=n$. The second situation arises for example for the Faraday $(n-2)$-form and the gauge potential 1-form in electromagnetism (see Example 4" in Section 4). However we were unable to find a general definition: this is left as a partially open problem. Regardless, note that this analysis shows that the right bracket 
(i.e., from the point of view adopted here) should have a definition which differs from those proposed in [21] and also from our previous definition in $[14]$.

In Section 5 we analyze the special case where the multisymplectic manifold is $\Lambda^{n} T^{*} \mathcal{N}$ : this example is important because it is the framework for Lepage-Dedecker theory. Note that this theory has been the subject of our companion paper [16]. We show that OF's and AOF's coincide on $\Lambda^{n} T^{*} \mathcal{N}$. This contrasts with the de Donder-Weyl theory in which - like all Lepage theories obtained by a restriction on a submanifold of $\Lambda^{n} T^{*} \mathcal{N}$ - the set of AOF's is a strict subset of the set of OF's. This singles out the LepageDedecker theory as being "complete": we say that $\Lambda^{n} T^{*} \mathcal{N}$ is pataplectic for quoting this property.

Another result in this paper is also motivated by the important example of $\Lambda^{n} T^{*} \mathcal{N}$, although it may have a larger range of application. In the Lepage-Dedecker theory indeed the Hamiltonian function and the Hamilton equations are invariant by deformations parallel to affine submanifolds called pseudofibers by Dedecker [6]. This sounds like something similar to a gauge invariance but the pseudofibers may intersect along singular sets, as already remarked by Dedecker [6]. In [16] we revisit this picture and proposed an intrinsic definition of this distribution which gives rise to a generalization that we call the generalized pseudofiber direction. We look here at the interplay of this notion with observable $(n-1)$-forms, namely showing in Paragraph 4.1.3 that - under some hypotheses - the resulting functional is invariant by deformation along the generalized pseudofibers directions.

A last question concerns the bracket between observable functionals obtained by integration of say $(n-1)$-forms on two different slices. This is a crucial question if one is concerned by the relativistic invariance of a symplectic theory. Indeed the only way to build a relativistic invariant theory of classical (or quantum) fields is to make sense of functionals (or observable operators) as defined on the set of solution (each one being a complete history in space-time), independently of the choice of a time coordinate. This requires at least that one should be able to define the bracket between say the observable functionals $\int_{\Sigma} F$ and $\int_{\widetilde{\Sigma}} G$ even when $\Sigma$ and $\widetilde{\Sigma}$ are different (imagine they correspond to two space-like hypersurfaces). One possibility for that is to assume that one of the two forms, say $F$ is such that $\int_{\Sigma} F$ depends uniquely on the homology class of $\Sigma$. Using Stoke's theorem one checks easily that such a condition is possible if $\{\mathcal{H}, F\}=0$. We call a $d y$ namical observable $(n-1)$-form any observable $(n-1)$-form which satisfies such a relation. All that leads us to the question of finding all such forms. 
This problem was investigated in [23] and discussed in [11] (in collaboration with S. Coleman). It led to an interesting but deceptive answer: for a linear variational problem (i.e., with a linear PDE, or for free fields) one can find a rich collection of dynamical OF's, roughly speaking in correspondence with the set of solutions of the linear PDE. However as soon as the problem becomes nonlinear (so for interacting fields) the set of dynamical OF's is much more reduced and corresponds to the symmetries of the problem (so it is in general finite dimensional). We come back here to this question in Section 6. We are looking at the example of a complex scalar field with one symmetry, so that the only dynamical OF's basically correspond to the total charge of the field. We show there that by a kind of Noether's procedure we can enlarge the set of dynamical OF's by including all smeared integrals of the current density. This example illustrates the fact that gauge symmetry helps strongly in constructing dynamical observable functionals. Another possibility in order to enlarge the number of dynamical functionals is when the nonlinear variational problem can be approximated by a linear one: this gives rise to observable functionals defined by expansions [12], [13].

As a conclusion we wish to insist about one of the main motivation for multisymplectic formalisms: it is to build a Hamiltonian theory which is consistent with the principles of Relativity, i.e., being covariant. Recall for instance that for all the multisymplectic formalisms which have been proposed one does not need to use a privilege time coordinate. But among them the Lepage-Dedecker is actually a quite natural framework in order to extend this democracy between space and time coordinates to the coordinates on fiber manifolds (i.e., along the fields themselves). This is quite in the spirit of the Kaluza-Klein theory and its modern avatars: 11-dimensional supergravity, string theory and M-theory. Indeed in the Dedecker theory, in contrast with the Donder-Weyl one, we do not need to split ${ }^{2}$ the variables into the horizontal (i.e., corresponding to space-time coordinates) and vertical (i.e., non horizontal) categories. Of course, as the reader can imagine, if we do not fix a priori the space-time/fields splitting, many new difficulties appear as for example: how to define forms which - in a non covariant way of thinking - should be of the type $d x^{\mu}$, where the $x^{\mu}$ 's are space-time coordinates, without a space-time background ${ }^{3}$ ? One possible way is by using

\footnotetext{
${ }^{2}$ Such a splitting has several drawbacks, for example it causes difficulties in order to define the stress-energy tensor.

${ }^{3}$ Another question which is probably related is: how to define a "slice", which plays the role of a constant time hypersurface without referring to a given space-time background? We propose in [16] a definition of such a slice which, roughly speaking, requires a slice to be transversal to all Hamiltonian $n$-curves, so that the dynamics only (i.e., the Hamiltonian function) should determine what are the slices. We give in [16] a characterization of these slices in the case where the multisymplectic manifold is $\Lambda^{n} T^{*} \mathcal{N}$.
} 
the (at first glance unpleasant) definition of copolarization given in Section 3 : the idea is that forms of the "type $d x^{\mu}$ " are defined collectively and each relatively to the other ones. We believe that this notion of copolarization corresponds somehow to the philosophy of general relativity: the observable quantities again are not measured directly, they are compared each to the other ones.

In exactly the same spirit we remark that the dynamical law (2) can be expressed in a slightly more general form which is: if $\Gamma$ is a Hamiltonian $n$-curve then

$$
\{\mathcal{H}, F\} d G_{\mid \Gamma}=\{\mathcal{H}, G\} d F_{\mid \Gamma},
$$

for all OF's $F$ and $G$ (see Proposition 3.1 and Theorem 3.1). Mathematically this is not much more difficult than (2). However (3) is more satisfactory from the point of view of relativity: no volume form $\omega$ is singled out, the dynamics just prescribe how to compare two observations.

\subsection{Notations}

The Kronecker symbol $\delta_{\nu}^{\mu}$ is equal to 1 if $\mu=\nu$ and equal to 0 otherwise. We shall also set

$$
\delta_{\nu_{1} \cdots \nu_{p}}^{\mu_{1} \cdots \mu_{p}}:=\left|\begin{array}{ccc}
\delta_{\nu_{1}}^{\mu_{1}} & \ldots & \delta_{\nu_{p}}^{\mu_{1}} \\
\vdots & & \vdots \\
\delta_{\nu_{1}}^{\mu_{p}} & \ldots & \delta_{\nu_{p}}^{\mu_{p}}
\end{array}\right| .
$$

In most examples, $\eta_{\mu \nu}$ is a constant metric tensor on $\mathbb{R}^{n}$ (which may be Euclidean or Minkowskian). The metric on his dual space his $\eta^{\mu \nu}$. Also, $\omega$ will often denote a volume form on some space-time: in local coordinates $\omega=d x^{1} \wedge \cdots \wedge d x^{n}$ and we will use several times the notation $\left.\omega_{\mu}:=\frac{\partial}{\partial x^{\mu}}\right\lrcorner \omega$, $\left.\omega_{\mu \nu}:=\frac{\partial}{\partial x^{\mu}} \wedge \frac{\partial}{\partial x^{\nu}}\right\lrcorner \omega$, etc. Partial derivatives $\frac{\partial}{\partial x^{\mu}}$ and $\frac{\partial}{\partial p_{\alpha_{1} \cdots \alpha_{n}}}$ will be sometime abbreviated by $\partial_{\mu}$ and $\partial^{\alpha_{1} \cdots \alpha_{n}}$ respectively.

When an index or a symbol is omitted in the middle of a sequence of

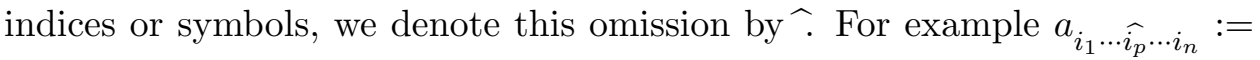
$a_{i_{1} \cdots i_{p-1} i_{p+1} \cdots i_{n}}, d x^{\alpha_{1}} \wedge \cdots \wedge \widehat{d x^{\alpha_{\mu}}} \wedge \cdots \wedge d x^{\alpha_{n}}:=d x^{\alpha_{1}} \wedge \cdots \wedge d x^{\alpha_{\mu-1}} \wedge d x^{\alpha_{\mu+1}} \wedge$ $\cdots \wedge d x^{\alpha_{n}}$.

If $\mathcal{N}$ is a manifold and $\mathcal{F} N$ a fiber bundle over $\mathcal{N}$, we denote by $\Gamma(\mathcal{N}, \mathcal{F} N)$ the set of smooth sections of $\mathcal{F} N$. Lastly we use the notations concerning the exterior algebra of multivectors and differential forms, following W.M. Tulczyjew [29]. If $\mathcal{N}$ is a differential $N$-dimensional manifold and $0 \leq k \leq$ $N, \Lambda^{k} T \mathcal{N}$ is the bundle over $\mathcal{N}$ of $k$-multivectors ( $k$-vectors in short) and $\Lambda^{k} T^{\star} \mathcal{N}$ is the bundle of differential forms of degree $k$ ( $k$-forms in short). 
Setting $\Lambda T \mathcal{N}:=\oplus_{k=0}^{N} \Lambda^{k} T \mathcal{N}$ and $\Lambda T^{\star} \mathcal{N}:=\oplus_{k=0}^{N} \Lambda^{k} T^{\star} \mathcal{N}$, there exists a unique duality evaluation map between $\Lambda T \mathcal{N}$ and $\Lambda T^{\star} \mathcal{N}$ such that for every decomposable $k$-vector field $X$, i.e., of the form $X=X_{1} \wedge \cdots \wedge X_{k}$, and for every $l$-form $\mu$, then $\langle X, \mu\rangle=\mu\left(X_{1}, \ldots, X_{k}\right)$ if $k=l$ and $=0$ otherwise. Then interior products $\lrcorner$ and $\llcorner$ are operations defined as follows. If $k \leq l$, the product $\lrcorner: \Gamma\left(\mathcal{N}, \Lambda^{k} T \mathcal{N}\right) \times \Gamma\left(\mathcal{N}, \Lambda^{l} T^{\star} \mathcal{N}\right) \longrightarrow \Gamma\left(\mathcal{N}, \Lambda^{l-k} T^{\star} \mathcal{N}\right)$ is given by

$$
\langle Y, X\lrcorner \mu\rangle=\langle X \wedge Y, \mu\rangle, \quad \forall(l-k) \text {-vector } Y .
$$

And if $k \geq l$, the product $\left\llcorner: \Gamma\left(\mathcal{N}, \Lambda^{k} T \mathcal{N}\right) \times \Gamma\left(\mathcal{N}, \Lambda^{l} T^{\star} \mathcal{N}\right) \longrightarrow\right.$ $\Gamma\left(\mathcal{N}, \Lambda^{k-l} T \mathcal{N}\right)$ is given by

$$
\langle X\llcorner\mu, \nu\rangle=\langle X, \mu \wedge \nu\rangle, \quad \forall(k-l) \text {-form } \nu .
$$

\section{Basic facts about multisymplectic manifolds}

We recall here the general framework introduced in [16].

\subsection{Multisymplectic manifolds}

Definition 2.1. Let $\mathcal{M}$ be a differential manifold. Let $n \in \mathbb{N}$ be some positive integer. A smooth $(n+1)$-form $\Omega$ on $\mathcal{M}$ is a multisymplectic form if and only if

(i) $\Omega$ is non degenerate, i.e., $\forall m \in \mathcal{M}, \forall \xi \in T_{m} \mathcal{M}$, if $\left.\xi\right\lrcorner \Omega_{m}=0$, then $\xi=0$

(ii) $\Omega$ is closed, i.e., $d \Omega=0$.

Any manifold $\mathcal{M}$ equipped with a multisymplectic form $\Omega$ will be called a multisymplectic manifold.

In the following, $N$ denotes the dimension of $\mathcal{M}$. For any $m \in \mathcal{M}$ we define the set

$$
D_{m}^{n} \mathcal{M}:=\left\{X_{1} \wedge \cdots \wedge X_{n} \in \Lambda^{n} T_{m} \mathcal{M} / X_{1}, \ldots, X_{n} \in T_{m} \mathcal{M}\right\}
$$

of decomposable $n$-vectors and denote by $D^{n} \mathcal{M}$ the associated bundle. 
Definition 2.2. Let $\mathcal{H}$ be a smooth real valued function defined over a multisymplectic manifold $(\mathcal{M}, \Omega)$. A Hamiltonian $n$-curve $\Gamma$ is a $n$-dimensional submanifold of $\mathcal{M}$ such that for any $m \in \Gamma$, there exists a $n$-vector $X$ in $\Lambda^{n} T_{m} \Gamma$ which satisfies

$$
X\lrcorner \Omega=(-1)^{n} d \mathcal{H} .
$$

We denote by $\mathcal{E}^{\mathcal{H}}$ the set of all such Hamiltonian $n$-curves. We also write for all $\left.m \in \mathcal{M},[X]_{m}^{\mathcal{H}}:=\left\{X \in D_{m}^{n} \mathcal{M} / X\right\lrcorner \Omega=(-1)^{n} d \mathcal{H}_{m}\right\}$.

Example 1 (The Lepage-Dedecker multisymplectic manifold $\left(\Lambda^{n} T^{*} \mathcal{N}, \Omega\right)$ ). It was studied in [16]. Here $\Omega:=d \theta$ where $\theta$ is the generalized Poincaré-Cartan 1-form defined by $\theta\left(X_{1}, \ldots X_{n}\right)=\left\langle\Pi^{*} X_{1}, \ldots, \Pi^{*} X_{n}, p\right\rangle$, $\forall X_{1}, \ldots, X_{n} \in T_{(q, p)}\left(\Lambda^{n} T^{*} \mathcal{N}\right)$ and $\Pi: \Lambda^{n} T^{*} \mathcal{N} \longrightarrow \mathcal{N}$ is the canonical projection. If we use local coordinates $\left(q^{\alpha}\right)_{1 \leq \alpha \leq n+k}$ on $\mathcal{N}$, then a basis of $\Lambda^{n} T_{q}^{*} \mathcal{N}$ is the family $\left(d q^{\alpha_{1}} \wedge \cdots \wedge d q^{\alpha_{n}}\right)_{1 \leq \alpha_{1}<\cdots<\alpha_{n} \leq n+k}$ and we denote by $p_{\alpha_{1} \cdots \alpha_{n}}$ the coordinates on $\Lambda^{n} T_{q}^{*} \mathcal{N}$ in this basis. Then $\Omega$ writes

$$
\Omega:=\sum_{1 \leq \alpha_{1}<\cdots<\alpha_{n} \leq n+k} d p_{\alpha_{1} \cdots \alpha_{n}} \wedge d q^{\alpha_{1}} \wedge \cdots \wedge d q^{\alpha_{n}} .
$$

If the Hamiltonian function $\mathcal{H}$ is associated to a Lagrangian variational problem on $n$-dimensional submanifolds of $\mathcal{N}$ by means of a Legendre correspondence (see [16], [6]) we then say that $\mathcal{H}$ is a Legendre image Hamiltonian function.

A particular case is when $\mathcal{N}=\mathcal{X} \times \mathcal{Y}$ where $\mathcal{X}$ and $\mathcal{Y}$ are manifolds of dimension $n$ and $k$ respectively. This situation occurs when we look at variational problems on maps $u: \mathcal{X} \longrightarrow \mathcal{Y}$. We denote by $q^{\mu}=x^{\mu}$, if $1 \leq \mu \leq n$, coordinates on $\mathcal{X}$ and by $q^{n+i}=y^{i}$, if $1 \leq i \leq k$, coordinates on $\mathcal{Y}$. We also denote by $e:=p_{1 \cdots n}, p_{i}^{\mu}:=p_{1 \cdots(\mu-1) i(\mu+1) \cdots n}$, $p_{i_{1} i_{2}}^{\mu_{1} \mu_{2}}:=p_{1 \cdots\left(\mu_{1}-1\right) i_{1}\left(\mu_{1}+1\right) \cdots\left(\mu_{2}-1\right) i_{2}\left(\mu_{2}+1\right) \cdots n}$, etc., so that

$$
\Omega=d e \wedge \omega+\sum_{j=1}^{n} \sum_{\mu_{1}<\cdots<\mu_{j}} \sum_{i_{1}<\cdots<i_{j}} d p_{i_{1} \cdots i_{j}}^{\mu_{1} \cdots \mu_{j}} \wedge \omega_{\mu_{1} \cdots \mu_{j}}^{i_{1} \cdots i_{j}},
$$

where, for $1 \leq p \leq n$,

$$
\begin{aligned}
\omega & :=d x^{1} \wedge \cdots \wedge d x^{n} \\
\omega_{\mu_{1} \cdots \mu_{p}}^{i_{1} \cdots i_{p}} & \left.:=d y^{i_{1}} \wedge \cdots \wedge d y^{i_{p}} \wedge\left(\frac{\partial}{\partial x^{\mu_{1}}} \wedge \cdots \wedge \frac{\partial}{\partial x^{\mu_{p}}}\right\lrcorner \omega\right) .
\end{aligned}
$$

Note that if $\mathcal{H}$ is the Legendre image of a Lagrangian action of the form $\int_{\mathcal{X}} \ell(x, u(x), d u(x)) \omega$ and if we denote by $p^{*}$ all coordinates $p_{i_{1} \cdots i_{j}}^{\mu_{1} \cdots \mu_{j}}$ for $j \geq 1$, we can always write $\mathcal{H}\left(q, e, p^{*}\right)=e+H\left(q, p^{*}\right)$ (see for instance [6], [14], [16]).

Other examples are provided by considering the restriction of $\Omega$ on any smooth submanifold of $\Lambda^{n} T^{*} \mathcal{N}$, like for instance the following. 
Example 2 (The de Donder-Weyl manifold $\mathcal{M}_{q}^{d D W}$ ). It is the submanifold of $\Lambda^{n} T_{q}^{*} \mathcal{N}$ defined by the constraints $p_{i_{1} \cdots i_{j}}^{\mu_{1} \cdots \mu_{j}}=0$, for all $j \geq 2$. We thus have

$$
\Omega^{d D W}=d e \wedge \omega+\sum_{\mu} \sum_{i} d p_{i}^{\mu} \wedge \omega_{\mu}^{i}
$$

Example 3 (The Palatini formulation of pure gravity in 4-dimensional space-time, see also [27]). We describe here the Riemannian (non Minkowskian) version of it. We consider $\mathbb{R}^{4}$ equipped with its standard metric $\eta_{I J}$ and with the standard volume 4 -form $\epsilon_{I J K L}$. Let $\mathfrak{p} \simeq$ $\left\{(a, v) \simeq\left(\begin{array}{cc}a & v \\ 0 & 1\end{array}\right) / a \in s o(4), v \in \mathbb{R}^{4}\right\} \simeq s o(4) \ltimes \mathbb{R}^{4}$ be the Lie algebra of the Poincaré group acting on $\mathbb{R}^{4}$. Now let $\mathcal{X}$ be a 4-dimensional manifold, the "space-time", and consider $\mathcal{M}:=\mathfrak{p} \otimes T^{*} \mathcal{X}$, the fiber bundle over $\mathcal{X}$ of 1 -forms with coefficients in $\mathfrak{p}$. We denote by $(x, e, A)$ a point in $\mathcal{M}$, where $x \in \mathcal{X}, e \in \mathbb{R}^{4} \otimes T_{x}^{*}$ and $A \in s o(4) \otimes T_{x}^{*}$. We shall work is the open subset of $\mathcal{M}$ where $e$ is rank 4 (so that the 4 components of $e$ define a coframe on $\left.T_{x} \mathcal{X}\right)$. First using the canonical projection $\Pi: \mathcal{M} \longrightarrow \mathcal{X}$ one can define a $\mathfrak{p}$-valued 1-form $\theta^{\mathfrak{p}}$ on $\mathcal{M}$ (similar to the Poincaré-Cartan 1-form) by

$$
\forall(x, e, A) \in \mathcal{M}, \forall X \in T_{(x, e, A)} \mathcal{X}, \quad \theta_{(x, e, A)}^{\mathfrak{p}}(X):=\left(e\left(\Pi^{*} X\right), A\left(\Pi^{*} X\right)\right) .
$$

Denoting (for $1 \leq I, J \leq 4)$ by $T^{I}: \mathfrak{p} \longrightarrow \mathbb{R},(a, v) \longmapsto v^{I}$ and by $R_{J}^{I}: \mathfrak{p} \longrightarrow$ $\mathbb{R},(a, v) \longmapsto a_{J}^{I}$, the coordinate mappings we can define a 4 -form on $\mathcal{M}$ by

$$
\begin{aligned}
\theta_{\text {Palatini }}:=\frac{1}{4 !} \epsilon_{I J K L} \eta^{L N}\left(T^{I} \circ \theta^{\mathfrak{p}}\right) \wedge & \left(T^{J} \circ \theta^{\mathfrak{p}}\right) \wedge \\
& \left(R_{N}^{K} \circ d \theta^{\mathfrak{p}}+\left(R_{M}^{K} \circ \theta^{\mathfrak{p}}\right) \wedge\left(R_{N}^{M} \circ \theta^{\mathfrak{p}}\right)\right) .
\end{aligned}
$$

Now consider any section of $\mathcal{M}$ over $\mathcal{X}$. Write it as $\Gamma:=\left\{\left(x, e_{x}, A_{x}\right) / x \in \mathcal{X}\right\}$ where now $e$ and $A$ are 1 -forms on $x$ (and not coordinates anymore). Then

$$
\int_{\Gamma} \theta_{\text {Palatini }}=\int_{\mathcal{X}} \frac{1}{4 !} \epsilon_{I J K L} \eta^{L N} e^{I} \wedge e^{J} \wedge F_{L}^{K},
$$

where $F_{J}^{I}:=d A_{J}^{I}+A_{K}^{I} \wedge A_{J}^{K}$ is the curvature of the connection 1-form $A$. We recognize the Palatini action for pure gravity in 4 dimensions: this functional has the property that a critical point of it provides us with a solution of Einstein gravity equation $R_{\mu \nu}-\frac{1}{2} g_{\mu \nu}=0$ by setting $g_{\mu \nu}:=\eta_{I J} e_{\mu}^{I} e_{\nu}^{J}$. By following the same steps as in the proof of Theorem 2.2 in [16] one proves that a 4-dimensional submanifold $\Gamma$ which is a critical point of this action, satisfies the Hamilton equation $X\lrcorner \Omega_{\text {Palatini }}=0$, where $\Omega_{\text {Palatini }}:=d \theta_{\text {Palatini }}$. Thus $\left(\mathcal{M}, \Omega_{\text {Palatini }}\right)$ is a multisymplectic manifold naturally associated to gravitation. In the above construction, by replacing $A$ and $F$ by their self-dual parts $A_{+}$and $F_{+}$(and so reducing the gauge group to $S O(3)$ ) one obtains the Ashtekar action. 
Remark also that a similar construction can be done for the Chern-Simon action in dimension 3 .

Definition 2.3. A symplectomorphism $\phi$ of a multisymplectic manifold $(\mathcal{M}, \Omega)$ is a smooth diffeomorphism $\phi: \mathcal{M} \longrightarrow \mathcal{M}$ such that $\phi^{*} \Omega=\Omega$. An infinitesimal symplectomorphism is a vector field $\xi \in \Gamma(\mathcal{M}, T \mathcal{M})$ such that $L_{\xi} \Omega=0$. We denote by $\mathfrak{s p}_{0} \mathcal{M}$ the set of infinitesimal symplectomorphisms of $(\mathcal{M}, \Omega)$.

Note that, since $\Omega$ is closed, $\left.L_{\xi} \Omega=d(\xi\lrcorner \Omega\right)$, so that a vector field $\xi$ belongs to $\mathfrak{s p}_{0} \mathcal{M}$ if and only if $\left.d(\xi\lrcorner \Omega\right)=0$. Hence if the homology group $H^{n}(\mathcal{M})$ is trivial there exists an $(n-1)$-form $F$ on $\mathcal{M}$ such that $d F+\xi\lrcorner \Omega=0$ : such an $F$ will be called an algebraic observable $(n-1)$ form (see Section 3.3).

\section{$2.2 \quad$ Pseudofibers}

It may happen that the dynamical structure encoded by the data of a multisymplectic manifold $(\mathcal{M}, \Omega)$ and a Hamiltonian function $\mathcal{H}$ is invariant by deformations along some particular submanifolds called pseudofibers. This situation is similar to gauge theory where two fields which are equivalent through a gauge transformation are supposed to correspond to the same physical state. A slight difference however lies in the fact that pseudofibers are not fibers in general and can intersect singularly. This arises for instance when $\mathcal{M}=\Lambda^{n} T^{*} \mathcal{N}$ and $\mathcal{H}$ is a Legendre image Hamiltonian function (see [6], [16]). In the latter situation the singular intersections of pseudofibers picture geometrically the constraints caused by gauge invariance. All that is the origin of the following definitions.

Definition 2.4. For all Hamiltonian function $\mathcal{H}: \mathcal{M} \longrightarrow \mathbb{R}$ and for all $m \in \mathcal{M}$ we define the generalized pseudofiber direction to be

$$
\begin{aligned}
L_{m}^{\mathcal{H}} & \left.:=\left\{\xi \in T_{m} \mathcal{M} / \forall X \in[X]_{m}^{\mathcal{H}}, \forall \delta X \in T_{X} D_{m}^{n} \mathcal{M},(\xi\lrcorner \Omega\right)(\delta X)=0\right\} \\
& \left.=\left(T_{[X]_{m}^{\mathcal{H}}} D_{m}^{n} \mathcal{M}\right\lrcorner \Omega\right)^{\perp} .
\end{aligned}
$$

And we write $L^{\mathcal{H}}:=\cup_{m \in \mathcal{M}} L_{m}^{\mathcal{H}} \subset T \mathcal{M}$ for the associated bundle.

Recall that $\left.[X]_{m}^{\mathcal{H}}:=\left\{X \in D_{m}^{n} \mathcal{M} / X\right\lrcorner \Omega=(-1)^{n} d \mathcal{H}_{m}\right\}$, where $D_{m}^{n} \mathcal{M}:=$ $\left\{X_{1} \wedge \cdots \wedge X_{n} \in \Lambda^{n} T_{m} \mathcal{M} / X_{1}, \ldots, X_{n} \in T_{m} \mathcal{M}\right\}$. Note that in the case where $\mathcal{M}=\Lambda^{n} T^{*} \mathcal{N}$ and $\mathcal{H}$ is the Legendre image Hamiltonian (see Section 2.1) then all generalized pseudofibers directions $L_{m}^{\mathcal{H}}$ are "vertical" i.e., $L_{m}^{\mathcal{H}} \subset$ $\operatorname{Ker} d \Pi_{m} \simeq \Lambda_{\Pi(m)}^{n} T^{*} \mathcal{N}$, where $\Pi: \Lambda^{n} T^{*} \mathcal{N} \longrightarrow \mathcal{N}$. 
Definition 2.5. We say that $\mathcal{H}$ is pataplectic invariant if

- $\forall \xi \in L_{m}^{\mathcal{H}}, d \mathcal{H}_{m}(\xi)=0$

- for all Hamiltonian $n$-curve $\Gamma \in \mathcal{E}^{\mathcal{H}}$, for all vector field $\xi$ which is a smooth section of $L^{\mathcal{H}}$, then, for $s \in \mathbb{R}$ sufficiently small, $\Gamma_{s}:=e^{s \xi}(\Gamma)$ is also a Hamiltonian $n$-curve.

We proved in [16] that, if $\mathcal{M}$ is an open subset of $\Lambda^{n} T^{*} \mathcal{N}$, any function on $\mathcal{M}$ which is a Legendre image Hamiltonian is pataplectic invariant.

\subsection{Functionals defined by means of integrations of forms}

An example of functional on $\mathcal{E}^{\mathcal{H}}$ is obtained by choosing a codimension $r$ submanifold $\Sigma$ of $\mathcal{M}$ (for $1 \leq r \leq n)$ and a $(p-1)$ )form $F$ on $\mathcal{M}$ with $r=n-p+1$ : it leads to the definition of

$$
\begin{aligned}
\int_{\Sigma} F: \mathcal{E}^{\mathcal{H}} & \longrightarrow \mathbb{R} \\
\Gamma & \longmapsto \int_{\Sigma \cap \Gamma} F
\end{aligned}
$$

But in order for this definition to be meaningful one should first make sure that the intersection $\Sigma \cap \Gamma$ is a $(p-1)$-dimensional submanifold. This is true if $\Sigma$ fulfills the following definition. (See also [16].)

Definition 2.6. Let $\mathcal{H}$ be a smooth real valued function defined over a multisymplectic manifold $(\mathcal{M}, \Omega)$. A slice of codimension $r$ is a cooriented submanifold $\Sigma$ of $\mathcal{M}$ of codimension $r$ such that for any $\Gamma \in \mathcal{E}^{\mathcal{H}}, \Sigma$ is transverse to $\Gamma$. By cooriented we mean that for each $m \in \Sigma$, the quotient space $T_{m} \mathcal{M} / T_{m} \Sigma$ is oriented continuously in function of $m$.

If we represent such a submanifold as a level set of a given function into $\mathbb{R}^{r}$ then it suffices that the restriction of such a function on any Hamiltonian $n$-curve have no critical point. We then say that the function is $r$-regular. In [16] we give a characterization of $r$-regular functions in $\Lambda^{n} T^{*} \mathcal{N}$.

A second question concerns then the choice of $F$ : what are the conditions on $F$ for $\int_{\Sigma} F$ to be a physically observable functional? Clearly the answer should agree with the experience of physicists, i.e., be based in the knowledge of all functionals which are physically meaningful. Our aim is here to understand which mathematical properties would characterize all such functionals. In the following we explore this question, by following two possible points of view. 


\section{The "dynamical" point of view}

\subsection{Observable $(n-1)$-forms}

We define here the concept of observable $(n-1)$-forms $F$. The idea is that given a point $m \in \mathcal{M}$ and a Hamiltonian function $\mathcal{H}$, if $X(m) \in[X]_{m}^{\mathcal{H}}$, then $\left\langle X(m), d F_{m}\right\rangle$ should not depend on the choice of $X(m)$ but only on $d \mathcal{H}_{m}$.

\subsubsection{Definitions}

Definition 3.1. Let $m \in \mathcal{M}$ and $a \in \Lambda^{n} T_{m}^{\star} \mathcal{M}$; $a$ is called a copolar $n$-form if and only if there exists an open dense subset $\mathcal{O}_{m}^{a} \mathcal{M} \subset D_{m}^{n} \mathcal{M}$ such that

$$
\left.\left.\forall X, \tilde{X} \in \mathcal{O}_{m}^{a} \mathcal{M}, \quad X\right\lrcorner \Omega=\tilde{X}\right\lrcorner \Omega \Longrightarrow a(X)=a(\tilde{X}) .
$$

We denote by $P_{m}^{n} T^{\star} \mathcal{M}$ the set of copolar $n$-forms at $m$. A $(n-1)$-form $F$ on $\mathcal{M}$ is called observable if and only if for every $m \in \mathcal{M}, d F_{m}$ is copolar i.e., $d F_{m} \in P_{m}^{n} T^{\star} \mathcal{M}$. We denote by $\mathfrak{P}^{n-1} \mathcal{M}$ the set of observable $(n-1)$-forms on $\mathcal{M}$.

Remark. For any $m \in \mathcal{M}, P^{n} T_{m}^{\star} \mathcal{M}$ is a vector space (in particular if $a, b \in P^{n} T_{m}^{\star} \mathcal{M}$ and $\lambda, \mu \in \mathbb{R}$ then $\lambda a+\nu b \in P^{n} T_{m}^{\star} \mathcal{M}$ and we can choose $\left.\mathcal{O}_{m}^{\lambda a+\nu b} \mathcal{M}=\mathcal{O}_{m}^{a} \mathcal{M} \cap \mathcal{O}_{m}^{b} \mathcal{M}\right)$ and so it is possible to construct a basis $\left(a_{1}, \ldots, a_{r}\right)$ for this space. Hence for any $a \in P^{n} T_{m}^{\star} \mathcal{M}$ we can write $a=$ $t^{1} a_{1}+\cdots+t^{r} a_{r}$ which implies that we can choose $\mathcal{O}_{m}^{a} \mathcal{M}=\cap_{s=1}^{r} \mathcal{O}_{m}^{a_{s}} \mathcal{M}$. So having chosen such a basis $\left(a_{1}, \ldots, a_{r}\right)$ we will denote by $\mathcal{O}_{m} \mathcal{M}:=$ $\cap_{s=1}^{r} \mathcal{O}_{m}^{a_{s}} \mathcal{M}$ (it is still open and dense in $D_{m}^{n} \mathcal{M}$ ) and in the following we will replace $\mathcal{O}_{m}^{a} \mathcal{M}$ by $\mathcal{O}_{m} \mathcal{M}$ in the above definition. We will also denote by $\mathcal{O} \mathcal{M}$ the associated bundle.

Lemma 3.1. Let $\phi: \mathcal{M} \longrightarrow \mathcal{M}$ be a symplectomorphism and $F \in \mathfrak{P}^{n-1} \mathcal{M}$. Then $\phi^{*} F \in \mathfrak{P}^{n-1} \mathcal{M}$. As a corollary, if $\xi \in \mathfrak{s p}_{0} \mathcal{M}$ (i.e., is an infinitesimal symplectomorphism) and $F \in \mathfrak{P}^{n-1} \mathcal{M}$, then $L_{\xi} F \in \mathfrak{P}^{n-1} \mathcal{M}$.

Proof. For any $n$-vector fields $X$ and $\tilde{X}$, which are sections of $\mathcal{O} M$, and for any $F \in \mathfrak{P}^{n-1} \mathcal{M}$,

$$
\left.\left.\left.X\lrcorner \Omega=\tilde{X}\lrcorner \Omega \Longleftrightarrow X\lrcorner \phi^{*} \Omega=\tilde{X}\right\lrcorner \phi^{*} \Omega \Longleftrightarrow\left(\phi_{*} X\right)\right\lrcorner \Omega=\left(\phi_{*} \widetilde{X}\right)\right\lrcorner \Omega
$$

implies

$$
\begin{aligned}
d F\left(\phi_{*} X\right)=d F\left(\phi_{*} \tilde{X}\right) \Longleftrightarrow \phi_{*} d F(X)=\phi_{*} d F(\tilde{X}) & \\
& \Longleftrightarrow d\left(\phi_{*} F\right)(X)=d\left(\phi_{*} F\right)(\tilde{X}) .
\end{aligned}
$$


Hence $\phi_{*} F \in \mathfrak{P}^{n-1} \mathcal{M}$.

Assume that a given Hamiltonian function $\mathcal{H}$ on $\mathcal{M}$ is such that $[X]_{m}^{\mathcal{H}} \subset$ $\mathcal{O}_{m} \mathcal{M}$. Then we shall say that $\mathcal{H}$ is admissible. If $\mathcal{H}$ is so, we define the pseudobracket for all observable $(n-1)$-form $F \in \mathfrak{P}^{n-1} \mathcal{M}$

$$
\{\mathcal{H}, F\}:=X\lrcorner d F=d F(X),
$$

where $X$ is any $n$-vector in $[X]_{m}^{\mathcal{H}}$. Remark that, using the same notations as in Example 1, if $\mathcal{M}=\Lambda^{n} T^{*}(\mathcal{X} \times \mathcal{Y})$ and $\mathcal{H}\left(x, u, e, p^{*}\right)=e+H\left(x, u, p^{*}\right)$, then $\left\{\mathcal{H}, x^{1} d x^{2} \wedge \cdots \wedge d x^{n}\right\}=1$.

\subsubsection{Dynamics equation using pseudobrackets}

Our purpose here is to generalize the classical well-known relation $d F / d t=$ $\{H, F\}$ of the classical mechanics.

Proposition 3.1. Let $\mathcal{H}$ be a smooth admissible Hamiltonian on $\mathcal{M}$ and $F$, $G$ two observable $(n-1)$-forms with $\mathcal{H}$. Then $\forall \Gamma \in \mathcal{E}^{\mathcal{H}}$,

$$
\{\mathcal{H}, F\} d G_{\mid \Gamma}=\{\mathcal{H}, G\} d F_{\mid \Gamma}
$$

Proof. This result is equivalent to proving that, if $X \in D_{m}^{n} \mathcal{M}$ is different of 0 and is tangent to $\Gamma$ at $m$, then

$$
\{\mathcal{H}, F\} d G(X)=\{\mathcal{H}, G\} d F(X) .
$$

Note that by rescaling, we can assume w.l.g. that $X\lrcorner \Omega=(-1)^{n} d \mathcal{H}$, i.e., $X \in[X]_{m}^{\mathcal{H}}$. But then (7) is equivalent to the obvious relation $\{\mathcal{H}, F\}\{\mathcal{H}, G\}$ $=\{\mathcal{H}, G\}\{\mathcal{H}, F\}$.

This result immediately implies the following result.

Corollary 3.1. Let $\mathcal{H}$ be a smooth admissible Hamiltonian function on $\mathcal{M}$. Assume that $F$ and $G$ are observable $(n-1)$-forms with $\mathcal{H}$ and that $\{\mathcal{H}, G\}=$ 1 (see the remark at the end of Paragraph 3.1.1). Then denoting $\omega:=d G$ we have:

$$
\forall \Gamma \in \mathcal{E}^{\mathcal{H}}, \quad\{\mathcal{H}, F\} \omega_{\mid \Gamma}=d F_{\mid \Gamma}
$$




\subsection{Observable $(p-1)$-forms}

We now introduce observable $(p-1)$-forms, for $1 \leq p<n$. The simplest situation where such forms play some role occurs when studying variational problems on maps $u: \mathcal{X} \longrightarrow \mathcal{Y}$ : any coordinate function $y^{i}$ on $\mathcal{Y}$ is an observable functional, which at least in a classical context can be measured. This observable 0 -form can be considered as canonically conjugate with the momentum observable form $\left.\partial / \partial y^{i}\right\lrcorner \theta$. A more complex situation is given by Maxwell equations: as proposed for the first time by I. Kanatchikov in [21] (see also [14]), the electromagnetic gauge potential and the Faraday fields can be modelled in an elegant way by observable 1 -forms and $(n-2)$-forms respectively.

Example 4 (Maxwell equations on Minkowski space-time). Assume here for simplicity that $\mathcal{X}$ is the four-dimensional Minkowski space. Then the gauge field is a 1-form $A(x)=A_{\mu}(x) d x^{\mu}$ defined over $\mathcal{X}$, i.e., a section of the bundle $T^{\star} \mathcal{X}$. The action functional in the presence of a (quadrivector) current field $j(x)=j^{\mu}(x) \partial / \partial x^{\mu}$ is $\int_{\mathcal{X}} l(x, A, d A) \omega$, where $\omega=d x^{0} \wedge d x^{1} \wedge$ $d x^{2} \wedge d x^{3}$ and

$$
l(x, A, d A)=-\frac{1}{4} F_{\mu \nu} F^{\mu \nu}-j^{\mu}(x) A_{\mu},
$$

where $F_{\mu \nu}:=\partial_{\mu} A_{\nu}-\partial_{\nu} A_{\mu}$ and $F^{\mu \nu}:=\eta^{\mu \lambda} \eta^{\nu \sigma} F_{\lambda \sigma}$ (see [14]). The associated multisymplectic manifold is then $\mathcal{M}:=\Lambda^{4} T^{\star}\left(T^{\star} \mathcal{X}\right)$ with the multisymplectic form

$$
\Omega=d e \wedge \omega+\sum_{\mu, \nu} d p^{A_{\mu} \nu} \wedge d a_{\mu} \wedge \omega_{\nu}+\cdots
$$

For simplicity we restrict ourself to the de Donder-Weyl submanifold (where all momentum coordinates excepted $e$ and $p^{A_{\mu} \nu}$ are set to 0$)$. This implies automatically the further constraints $p^{A_{\mu} \nu}+p^{A_{\nu} \mu}=0$, because the Legendre correspondence degenerates when restricted to the de Donder-Weyl submanifold. We shall hence denote

$$
p^{\mu \nu}:=p^{A_{\mu} \nu}=-p^{A_{\nu} \mu} .
$$

Let us call $\mathcal{M}^{\text {Max }}$ the resulting multisymplectic manifold. Then the multisymplectic form can be written as

$$
\Omega=d e \wedge \omega+d \pi \wedge d a \quad \text { where } a:=a_{\mu} d x^{\mu} \text { and } \pi:=-\frac{1}{2} \sum_{\mu, \nu} p^{\mu \nu} \omega_{\mu \nu} .
$$

(We also have $d \pi \wedge d a=\sum_{\mu, \nu} d p^{\mu \nu} \wedge d a_{\mu} \wedge \omega_{\nu}$.) Note that here $a_{\mu}$ is not anymore a function of $x$ but a fiber coordinate. The Hamiltonian is then

$$
\mathcal{H}(x, a, p)=e-\frac{1}{4} \eta_{\mu \lambda} \eta_{\nu \sigma} p^{\mu \nu} p^{\lambda \sigma}+j^{\mu}(x) a_{\mu} .
$$




\subsubsection{Copolarization and polarization}

The dynamical properties of $(p-1)$-forms are more subtle for $1 \leq p<n$ than for $p=n$, since if $F$ is such a $(p-1)$-form then there is no way a priori to "evaluate" $d F$ along a Hamiltonian $n$-vector $X$ and a fortiori no way to make sense that " $d F_{\mid X}$ should not depend on $X$ but on $d \mathcal{H}_{m}$ ". This situation is in some sense connected with the problem of measuring a distance in relativity: we actually never measure the distance between two points (finitely or infinitely close) but we do compare observable quantities (distance, time) between themselves. This analogy suggests us the conclusion that we should define observable $(p-1)$-forms collectively. The idea is naively that if for instance $F_{1}, \ldots, F_{n}$ are 0 -forms, then they are observable forms if $d F_{1} \wedge \cdots \wedge d F_{n}$ can be "evaluated" in the sense that $d F_{1} \wedge \cdots \wedge d F_{n}(X)$ does not depend on the choice of the Hamiltonian $n$-vector $X$ but on $d \mathcal{H}$. So it just means that $d F_{1} \wedge \cdots \wedge d F_{n}$ is copolar. Henceforth, this idea will be assumed in the following definitions.

Definition 3.2. Let $\mathcal{M}$ be a multisymplectic manifold. A copolarization on $\mathcal{M}$ is a smooth vector subbundle denoted by $P^{*} T^{\star} \mathcal{M}$ of $\Lambda^{*} T^{\star} \mathcal{M}$ satisfying the following properties

- $P^{*} T^{\star} \mathcal{M}:=\oplus_{j=1}^{N} P^{j} T^{\star} \mathcal{M}$, where $P^{j} T^{\star} \mathcal{M}$ is a subbundle of $\Lambda^{j} T^{\star} \mathcal{M}$

- for each $m \in \mathcal{M},\left(P^{*} T_{m}^{\star} \mathcal{M},+, \wedge\right)$ is a subalgebra of $\left(\Lambda^{*} T_{m}^{\star} \mathcal{M},+, \wedge\right)$

- $\forall m \in \mathcal{M}$ and $\forall a \in \Lambda^{n} T_{m}^{\star} \mathcal{M}, a \in P^{n} T_{m}^{\star} \mathcal{M}$ if and only if $\forall X, \widetilde{X} \in \mathcal{O}_{m}$, $X\lrcorner \Omega=\widetilde{X}\lrcorner \Omega \Longrightarrow a(X)=a(\widetilde{X})$.

Definition 3.3. Let $\mathcal{M}$ be a multisymplectic manifold with a copolarization $P^{*} T^{\star} \mathcal{M}$. Then for $1 \leq p \leq n$, the set of observable $(p-1)$-forms associated to $P^{*} T^{\star} \mathcal{M}$ is the set of smooth $(p-1)$-forms $F$ (sections of $\Lambda^{p-1} T^{\star} \mathcal{M}$ ) such that for any $m \in \mathcal{M}, d F_{m} \in P^{p} T_{m}^{\star} \mathcal{M}$. This set is denoted by $\mathfrak{P}^{p-1} \mathcal{M}$. We shall write $\mathfrak{P}^{*} \mathcal{M}:=\oplus_{p=1}^{n} \mathfrak{P}^{p-1} \mathcal{M}$.

Definition 3.4. Let $\mathcal{M}$ be a multisymplectic manifold with a copolarization $P^{*} T^{\star} \mathcal{M}$. For each $m \in \mathcal{M}$ and $1 \leq p \leq n$, consider the equivalence relation in $\Lambda^{p} T_{m} \mathcal{M}$ defined by $X \sim \widetilde{X}$ if and only if $\langle X, a\rangle=\langle\widetilde{X}, a\rangle, \forall a \in P^{p} T_{m}^{\star} \mathcal{M}$. Then the quotient set $P^{p} T_{m} \mathcal{M}:=\Lambda^{p} T_{m} \mathcal{M} / \sim$ is called a polarization of $\mathcal{M}$. If $X \in \Lambda^{p} T_{m} \mathcal{M}$, we denote by $[X] \in P^{p} T_{m} \mathcal{M}$ its equivalence class.

Equivalently a polarization can be defined as being the dual bundle of the copolarization $P^{*} T^{\star} \mathcal{M}$. 


\subsubsection{Examples of copolarization}

On an open subset $\mathcal{M}$ of $\Lambda^{n} T^{*} \mathcal{N}$ we can construct the following copolarization, that we will call standard: for each $(q, p) \in \Lambda^{n} T^{*} \mathcal{N}$ and for $1 \leq p \leq n-1$ we take $P_{(q, p)}^{p} T^{*} \mathcal{M}$ to be the vector space spanned by $\left(d q^{\alpha_{1}} \wedge \cdots \wedge d q^{\alpha_{p}}\right)_{1 \leq \alpha_{1}<\cdots<\alpha_{p} \leq n+k} ;$ and $\left.P_{(q, p)}^{n} T^{*} \mathcal{M}=\{\xi\lrcorner \Omega / \xi \in T_{m} \mathcal{M}\right\}$. It means that $P_{(q, p)}^{n} T^{*} \mathcal{M}$ contains all $d q^{\alpha_{1}} \wedge \cdots \wedge d q^{\alpha_{n}}$ 's plus forms of the type $\xi\lrcorner \Omega$, for $\xi \in T_{q} \mathcal{N}$ (which corresponds to differentials of momentum and energy-momentum observable $(n-1)$-forms).

Another situation is the following.

Example $4^{\prime}$ (Maxwell equations). We continue Example 4 given at the beginning of this Section. In $\mathcal{M}^{\text {Max }}$ with the multisymplectic form $\Omega=$ $d e \wedge \omega+d \pi \wedge d a$ the more natural choice of copolarization is:

$$
\begin{aligned}
& \text { - } P_{(q, p)}^{1} T^{*} \mathcal{M}^{\mathrm{Max}}=\bigoplus_{0 \leq \mu \leq 3} \mathbb{R} d x^{\mu} \\
& \text { - } P_{(q, p)}^{2} T^{*} \mathcal{M}^{\mathrm{Max}}=\bigoplus_{0 \leq \mu_{1}<\mu_{2} \leq 3} \mathbb{R} d x^{\mu_{1}} \wedge d x^{\mu_{2}} \oplus \mathbb{R} d a \text {, where } d a:= \\
& \sum_{\mu=0}^{3} d a_{\mu} \wedge d x^{\mu} \\
& \text { - } P_{(q, p)}^{3} T^{*} \mathcal{M}^{\mathrm{Max}}=\bigoplus_{0 \leq \mu_{1}<\mu_{2}<\mu_{3} \leq 3} \mathbb{R} d x^{\mu_{1}} \wedge d x^{\mu_{2}} \wedge d x^{\mu_{3}} \oplus \bigoplus_{0 \leq \mu \leq 3} \mathbb{R} d x^{\mu} \wedge d a \oplus \\
& \mathbb{R} d \pi . \\
& \text { - } P_{(q, p)}^{4} T^{*} \mathcal{M}^{\mathrm{Max}}=\mathbb{R} \omega \oplus \bigoplus_{0 \leq \mu_{1}<\mu_{2} \leq 3} \mathbb{R} d x^{\mu_{1}} \wedge d x^{\mu_{2}} \wedge d a \oplus \bigoplus_{0 \leq \mu \leq 3} \mathbb{R} d x^{\mu} \wedge d \pi \oplus \\
& \left.\bigoplus_{0 \leq \mu \leq 3} \mathbb{R} \frac{\partial}{\partial x^{\mu}}\right\lrcorner \theta
\end{aligned}
$$

It is worth stressing out the fact that we did not include the differential of the coordinates $a_{\mu}$ of $a$ in $P_{(q, p)}^{1} T^{*} \mathcal{M}^{\mathrm{Max}}$. There are strong physical reasons for that since the gauge potential is not observable. But another reason is that if we had included the $d a_{\mu}$ 's in $P_{(q, p)}^{1} T^{*} \mathcal{M}^{\text {Max }}$, we would not have a copolarization since $d a_{\mu} \wedge d \pi$ does not satisfy the condition $\forall X, \widetilde{X} \in \mathcal{O}_{m}$, $[X]=[\widetilde{X}] \Rightarrow b(X)=b(\widetilde{X})$ required. This confirms the agreement of the definition of copolarization with physical purposes. 


\subsubsection{Results on the dynamics}

We wish here to generalize Proposition 3.1 to observable $(p-1)$-forms for $1 \leq p<n$. This result actually justifies the relevance of Definitions 3.2, 3.3 and 3.4. Throughout this section we assume that $(\mathcal{M}, \Omega)$ is equipped with a copolarization. We start with some technical results. If $\mathcal{H}$ is a Hamiltonian function, we recall that we denote by $[X]^{\mathcal{H}}$ the class modulo $\sim$ of decomposable $n$-vector fields $X$ such that $X\lrcorner \Omega=(-1)^{n} d \mathcal{H}$.

Lemma 3.2. Let $X$ and $\widetilde{X}$ be two decomposable n-vectors in $D_{m}^{n} \mathcal{M}$. If $X \sim \widetilde{X}$ then $\forall 1 \leq p \leq n, \forall a \in P^{p} T_{m}^{\star} \mathcal{M}$,

$$
X\llcorner a \sim \tilde{X}\llcorner a .
$$

Hence we can define $[X]\left\llcorner a:=\left[X\llcorner a] \in P^{n-p} T \mathcal{M}\right.\right.$.

Proof. This result amounts to the property that for all $0 \leq p \leq n, \forall a \in$ $P^{p} T_{m}^{\star} \mathcal{M}, \forall b \in P^{n-p} T_{m}^{\star} \mathcal{M}$

$$
\langle X\llcorner a, b\rangle=\langle\widetilde{X}\llcorner a, b\rangle \quad \Longleftrightarrow a \wedge b(X)=a \wedge b(\tilde{X}),
$$

which is true because of $[X]=[\widetilde{X}]$ and $a \wedge b \in P^{n} T_{m}^{\star} \mathcal{M}$.

As a consequence of Lemma 3.2, we have the following definition.

Definition 3.5. Let $F \in \mathfrak{P}^{p-1} \mathcal{M}$ and $\mathcal{H}$ a Hamiltonian function. The pseudobracket $\{\mathcal{H}, F\}$ is the section of $P^{n-p} T \mathcal{M}$ defined by

$$
\{\mathcal{H}, F\}:=(-1)^{(n-p) p}[X]^{\mathcal{H}}\llcorner d F .
$$

In case $p=n,\{\mathcal{H}, F\}$ is just the scalar function $\left.[X]^{\mathcal{H}}\right\lrcorner d F=\left\langle[X]^{\mathcal{H}}, d F\right\rangle$.

We now prove the basic result relating this notion to the dynamics.

Theorem 3.1. Let $(\mathcal{M}, \Omega)$ be a multisymplectic manifold. Assume that $1 \leq p \leq n, 1 \leq q \leq n$ and $n \leq p+q$. Let $F \in \mathfrak{P}^{p-1} \mathcal{M}$ and $G \in \mathfrak{P}^{q-1} \mathcal{M}$. Let $\Sigma$ be a slice of codimension $2 n-p-q$ and $\Gamma$ a Hamiltonian $n$-curve. Then for any $(p+q-n)$-vector $Y$ tangent to $\Sigma \cap \Gamma$, we have

$$
\left.\{\mathcal{H}, F\}\lrcorner d G(Y)=(-1)^{(n-p)(n-q)}\{\mathcal{H}, G\}\right\lrcorner d F(Y),
$$

which is equivalent to

$$
\left.\{\mathcal{H}, F\}\lrcorner d G_{\mid \Gamma}=(-1)^{(n-p)(n-q)}\{\mathcal{H}, G\}\right\lrcorner d F_{\mid \Gamma} .
$$


Proof. Proving (9) is equivalent to proving

$$
\langle\{\mathcal{H}, F\} \wedge Y, d G\rangle=(-1)^{(n-p)(n-q)}\langle\{\mathcal{H}, G\} \wedge Y, d F\rangle
$$

We thus need to compute first $\{\mathcal{H}, F\} \wedge Y$. For that purpose, we use Definition 3.5: $\{\mathcal{H}, F\}=(-1)^{(n-p)(n-q)}[X]^{\mathcal{H}}\llcorner d F$. Of course it will be more suitable to use the representant of $[X]^{\mathcal{H}}$ which is tangent to $\Gamma$ : we let $\left(X_{1}, \ldots, X_{n}\right)$ to be a basis of $T_{m} \Gamma$ such that

$$
X_{1} \wedge \cdots \wedge X_{n}=: X \in[X]^{\mathcal{H}} .
$$

Then we can write

$$
Y=\sum_{\nu_{1}<\cdots<\nu_{p+q-n}} T^{\nu_{1} \cdots \nu_{p+q-n}} X_{\nu_{1}} \wedge \cdots \wedge X_{\nu_{p+q-n}} .
$$

Now

$$
\begin{aligned}
\{\mathcal{H}, F\} & =(-1)^{(n-p) p}[X]^{\mathcal{H}}\llcorner d F \\
& =(-1)^{(n-p) p} \sum_{\substack{\mu_{1}<\cdots<\mu_{p} \\
\mu_{p+1}<\cdots<\mu_{n}}} \delta_{1 \cdots n}^{\mu_{1} \cdots \mu_{n}} d F\left(X_{\mu_{1}}, \ldots, X_{\mu_{p}}\right) X_{\mu_{p+1}} \wedge \cdots \wedge X_{\mu_{n}},
\end{aligned}
$$

so that

$$
\begin{aligned}
& \{\mathcal{H}, F\} \wedge Y=(-1)^{(n-p)(p+q-n)} Y \wedge\{\mathcal{H}, F\} \\
& =(-1)^{(n-p)(n-q)} \sum_{\nu_{1}<\cdots<\nu_{p+q-n}} \sum_{\substack{\mu_{1}<\cdots<\mu_{p} \\
\mu_{p+1}<\cdots<\mu_{n}}} T^{\nu_{1} \cdots \nu_{p+q-n}} \delta_{1 \cdots n}^{\mu_{1} \cdots \mu_{n}} \\
& \quad d F\left(X_{\mu_{1}}, \ldots, X_{\mu_{p}}\right) X_{\nu_{1}} \wedge \cdots \wedge X_{\nu_{p+q-n}} \wedge X_{\mu_{p+1}} \wedge \cdots \wedge X_{\mu_{n}} .
\end{aligned}
$$

Now $X_{\nu_{1}} \wedge \cdots \wedge X_{\nu_{p+q-n}} \wedge X_{\mu_{p+1}} \wedge \cdots \wedge X_{\mu_{n}} \neq 0$ if and only if it is possible to complete the family $\left\{X_{\nu_{1}}, \ldots, X_{\nu_{p+q-n}}\right\}$ by $\left\{X_{\lambda_{1}}, \ldots, X_{\lambda_{n-q}}\right\}$ in such a way that $\left\{X_{\nu_{1}}, \ldots, X_{\nu_{p+q-n}}, X_{\lambda_{1}}, \ldots, X_{\lambda_{n-q}}\right\}=\left\{X_{\mu_{1}}, \ldots, X_{\mu_{p}}\right\}$ and $\delta_{\mu_{1} \cdots \mu_{p}}^{\nu_{1} \cdots \nu_{p+q-n} \lambda_{1} \cdots \lambda_{n-q}} \neq 0$. Hence

$\{\mathcal{H}, F\} \wedge Y$

$$
\begin{aligned}
& =(-1)^{(n-p)(n-q)} \sum_{\substack{\mu_{1}<\cdots<\mu_{p} \\
\mu_{p+1}<\cdots<\mu_{n}}} \sum_{\substack{\nu_{1}<\cdots<\nu_{p+q-n} \\
\lambda_{1}<\cdots<\lambda_{n-q}}} \delta_{\mu_{1} \cdots \mu_{p}}^{\nu_{1} \cdots \nu_{p+q-n} \lambda_{1} \cdots \lambda_{n-q}} T^{\nu_{1} \cdots \nu_{p+q-n}} \delta_{1 \cdots n}^{\mu_{1} \ldots \mu_{n}} \\
& d F\left(X_{\nu_{1}}, \ldots, X_{\nu_{p+q-n}}, X_{\lambda_{1}}, \ldots, X_{\lambda_{n-q}}\right) X_{\nu_{1}} \wedge \cdots \wedge X_{\nu_{p+q-n}} \wedge X_{\mu_{p+1}} \wedge \cdots \wedge X_{\mu_{n}}
\end{aligned}
$$




$$
\begin{aligned}
& =(-1)^{(n-p)(n-q)} \sum_{\substack{\mu_{1}<\cdots<\mu_{p} \\
\mu_{p+1}<\cdots<\mu_{n}}} \sum_{\substack{\nu_{1}<\cdots<\nu_{p+q-n} \\
\lambda_{1}<\cdots<\lambda_{n-q}}} \delta_{1 \cdots n}^{\nu_{1} \cdots \nu_{p+q-n} \lambda_{1} \cdots \lambda_{n-q} \mu_{p+1} \cdots \mu_{n}} T^{\nu_{1} \cdots \nu_{p+q-n}} \\
& \cdot\left(X_{\nu_{1}} \wedge \cdots \wedge X_{\nu_{p+q-n}}-d F\right)\left(X_{\lambda_{1}}, \ldots, X_{\lambda_{n-q}}\right) \\
& \cdot X_{\nu_{1}} \wedge \cdots \wedge X_{\nu_{p+q-n}} \wedge X_{\mu_{p+1}} \wedge \cdots \wedge X_{\mu_{n}} \\
& =(-1)^{(n-p)(n-q)}(-1)^{(n-q)(p+q-n)} X\llcorner(Y\lrcorner d F) \\
& =(-1)^{(n-q) q} X\left\llcorner\left(Y \_d F\right)\right. \text {. }
\end{aligned}
$$

We conclude that

$$
\begin{aligned}
\langle\{\mathcal{H}, F\} \wedge Y, d G\rangle & \left.=(-1)^{(n-q) q}\langle X,(Y\lrcorner d F) \wedge d G\right\rangle \\
& =\langle X, d G \wedge(Y\lrcorner d F)\rangle \\
& =\langle X\llcorner d G, Y\lrcorner d F\rangle \\
& \left.=(-1)^{(n-q) q}\langle\{\mathcal{H}, G\}, Y\lrcorner d F\right\rangle \\
& =(-1)^{(n-q)(n-p)}\langle\{\mathcal{H}, G\} \wedge Y, d F\rangle .
\end{aligned}
$$

So the result follows.

Corollary 3.2. Assume the same hypothesis as in Theorem 3.1, then we have the following relations (by decreasing the generality)

(i) If $F \in \mathfrak{P}^{p-1} \mathcal{M}$ and $G \in \mathfrak{P}^{n-1} \mathcal{M}$, then

$$
\{\mathcal{H}, F\}\lrcorner d G_{\mid \Gamma}=\{\mathcal{H}, G\} d F_{\mid \Gamma}
$$

(ii) If $F \in \mathfrak{P}^{p-1} \mathcal{M}$ and if $G \in \mathfrak{P}^{n-1} \mathcal{M}$ is such that $\{\mathcal{H}, G\}=1$, then denoting by $\omega:=d G$ ( $a$ "volume form")

$$
\{\mathcal{H}, F\}\lrcorner \omega_{\mid \Gamma}=d F_{\mid \Gamma} .
$$

(iii) If $F, G \in \mathfrak{P}^{n-1} \mathcal{M}$, we recover proposition 1 .

Proof. It is a straightforward application of Theorem 3.1.

Example 5 (Consider a variational problem on maps $u: \mathcal{X} \longrightarrow \mathcal{Y}$ as in Example 2, Section 2.2.1). Take $F=y^{i}$ (a 0 -form) and $G=x^{1} d x^{2} \wedge$ $\cdots \wedge d x^{n}$, in such a way that $d G=\omega$, the volume form. Then we are in case (ii) of the corollary: we can compute that $\left.\left\{\mathcal{H}, y^{i}\right\}\right\lrcorner \omega=\sum_{\mu} \partial \mathcal{H} / \partial p_{i}^{\mu} d x^{\mu}$ and $\{\mathcal{H}, G\} d y^{i}=d y^{i}$. Hence this implies the relation $d y_{\mid \Gamma}^{i}=\sum_{\mu} \partial \mathcal{H} / \partial p_{i}^{\mu} d x_{\mid \Gamma}^{\mu}$. 


\section{The "symmetry" point of view}

An alternative way to define "observable forms" is to suppose that they are related to infinitesimal symplectomorphisms in a way analogous to the situation in classical mechanics. This point of view is more directly related to symmetries and Noether's theorem, since one can anticipate (correctly) that if the Hamiltonian function $\mathcal{H}$ is invariant by an infinitesimal symplectomorphism then the corresponding observable form is closed, thus recovering a divergence free vector field. The advantages of this definition are that we are able to define a notion of Poisson bracket between such observable forms easily and that this Poisson bracket is directly related to the one used by physicists for quantizing fields.

\subsection{Algebraic observable $(n-1)$-forms}

\subsubsection{Definitions}

Definition 4.1. Let $m \in \mathcal{M}$ and $a \in \Lambda^{n} T_{m}^{\star} \mathcal{M} ; a$ is called algebraic copolar if and only if there exists a unique $\xi \in T_{m} \mathcal{M}$ such that $\left.a+\xi\right\lrcorner \Omega=0$. We denote by $P_{0}^{n} T_{m}^{\star} \mathcal{M}$ the set of algebraic copolar $n$-forms.

A $(n-1)$-form $F$ on $(\mathcal{M}, \Omega)$ is called algebraic observable $(n-1)$-form if and only if for all $m \in \mathcal{M}, d F_{m} \in P_{0}^{n} T_{m}^{\star} \mathcal{M}$. We denote by $\mathfrak{P}_{0}^{n-1} \mathcal{M}$ the set of all algebraic observable $(n-1)$-forms.

In other words a $(n-1)$-form $F$ is algebraic observable if and only if there exists a vector field $\xi$ satisfying $d F+\xi\lrcorner \Omega=0$. Then we denote by $\xi_{F}$ this unique vector field. A straightforward observation is that any algebraic observable $(n-1)$-form satisfies automatically property (6) and so is an observable $(n-1)$-form. Actually the following Lemma implies that algebraic observable $(n-1)$-form are characterized by a property similar to (6) but stronger. (Indeed $\mathcal{O}^{n} \mathcal{M} \subset D^{n} \mathcal{M}$ is a submanifold of $\Lambda^{n} T_{m}^{\star} \mathcal{M}$.)

Lemma 4.1. Let $m \in \mathcal{M}$ and $\phi \in \Lambda_{m}^{n} T_{m}^{\star} \mathcal{M}$. Then $\phi \in P_{0}^{n} T_{m}^{\star} \mathcal{M}$ if and only if

$$
\left.\left.\forall X, \tilde{X} \in \Lambda^{n} T_{m} \mathcal{M}, \quad X\right\lrcorner \Omega=\widetilde{X}\right\lrcorner \Omega \quad \Longrightarrow \quad \phi(X)=\phi(\tilde{X}) .
$$

Proof. Let us fix some point $m \in \mathcal{M}$ and let $\phi \in \Lambda_{m}^{n} T_{m}^{\star} \mathcal{M}$. We consider the two following linear maps

$$
\begin{aligned}
& L: \Lambda^{n} T_{m} \mathcal{M} \longrightarrow T_{m}^{*} \mathcal{M} \quad \text { and } \quad K: T_{m} \mathcal{M} \longrightarrow\left(\Lambda^{n} T_{m} \mathcal{M}\right)^{*} \\
& \left.\left.X \quad \longmapsto(-1)^{n} X\right\lrcorner \Omega \quad \xi \longmapsto \xi\right\lrcorner \Omega \text {, }
\end{aligned}
$$


where we used the identification $\left(\Lambda^{n} T_{m} \mathcal{M}\right)^{*} \simeq \Lambda^{n} T_{m}^{*} \mathcal{M}$ for defining $K$. We first observe that $K$ is the adjoint of $L$. Indeed

$$
\begin{gathered}
\forall X \in \Lambda^{n} T_{m} \mathcal{M}, \forall \xi \in T_{m} \mathcal{M}, \\
\left.\langle X, K(\xi)\rangle=\xi\lrcorner \Omega(X)=(-1)^{n} X\right\lrcorner \Omega(\xi)=\langle\xi, L(X)\rangle .
\end{gathered}
$$

Hence since $K$ is one to one (because $\Omega$ is non degenerate) $L$ is onto. Moreover we can consider the following maps induced by $L$ and $K$ : $[L]$ : $\Lambda^{n} T_{m} \mathcal{M} / \operatorname{Ker} L \longrightarrow T_{m}^{*} \mathcal{M}$ and $[K]: T_{m} \mathcal{M} \longrightarrow\left(\Lambda^{n} T_{m} \mathcal{M} / \operatorname{Ker} L\right)^{*}$. Again $[K]=[L]^{*}$ and the fact that $L$ is onto implies that $[L]$ is a vector space isomorphism and that $[K]$ is so.

Now observe that the set of $\phi \in \Lambda^{n} T_{m}^{*} \mathcal{M}$ which satisfies (11) coincides with $\left(\Lambda^{n} T_{m} \mathcal{M} / \operatorname{Ker} L\right)^{*}$. Hence the conclusion of the Lemma follows from the fact that $[K]: T_{m} \mathcal{M} \longrightarrow\left(\Lambda^{n} T_{m} \mathcal{M} / \operatorname{Ker} L\right)^{*}$ is an isomorphism.

Hence $\mathfrak{P}_{0}^{n-1} \mathcal{M} \subset \mathfrak{P}^{n-1} \mathcal{M}$. We wish to single out multisymplectic manifolds where this inclusion is an identity:

Definition 4.2. A multisymplectic manifold $(\mathcal{M}, \Omega)$ is pataplectic if and only if the set of observable $(n-1)$-forms coincides with the set of algebraic observable $(n-1)$-forms, i.e., $\mathfrak{P}_{0}^{n-1} \mathcal{M}=\mathfrak{P}^{n-1} \mathcal{M}$.

We will see in the next paragraph that the multisymplectic manifold corresponding to the de Donder-Weyl theory is not pataplectic (if $k \geq 2$ ). But any open subset of $\Lambda^{n} T^{*} \mathcal{N}$ is pataplectic, as proved in Section 5 (there we also characterize completely the set of algebraic observable $(n-1)$-forms).

\subsubsection{Example of observable $(n-1)$-forms which are not algebraic observable $(n-1)$-forms}

In order to picture the difference between algebraic and non algebraic observable $(n-1)$-forms, let us consider the example of the de Donder-Weyl theory here corresponding to a submanifold of $\mathcal{M}=\Lambda^{n} T^{\star}\left(\mathbb{R}^{n} \times \mathbb{R}^{k}\right.$ ) (for $n, k \geq 2$ ) defined in Example 2. We use the same notations as in Example 2. It is easy to see that the set $\mathfrak{P}_{0}^{n-1} \mathcal{M}^{d D W}$ of algebraic observable $(n-1)$ forms coincides with the set of $(n-1)$-forms $F$ on $\mathcal{M}^{d D W}$ such that, at each point $m \in \mathcal{M}^{d D W}, d F_{m}$ has the form

$$
\left.d F_{m}=\left(a^{\mu} \frac{\partial}{\partial x^{\mu}}+b^{i} \frac{\partial}{\partial y^{i}}\right)\right\lrcorner \Omega+f \omega+f_{i}^{\mu} \omega_{\mu}^{i}
$$


(where we assume summation over all repeated indices). Now we observe that, by the Plücker relations,

$$
\begin{gathered}
\forall 1 \leq p \leq n, \forall X \in D^{n} \mathcal{M}^{d D W}, \\
(\omega(X))^{p-1} \omega_{\mu_{1} \cdots \mu_{p}}^{i_{1} \cdots i_{p}}(X)=\operatorname{det}\left(\omega_{\mu_{\alpha}}^{i_{\beta}}(X)\right)_{1 \leq \alpha, \beta \leq p},
\end{gathered}
$$

so it turns out that, if $X \in D^{n} \mathcal{M}^{d D W}$ is such that $\omega(X) \neq 0$, then all the values $\omega_{\mu_{1} \cdots \mu_{p}}^{i_{1} \cdots i_{p}}(X)$ can be computed from $\omega(X)$ and $\left(\omega_{\mu}^{i}(X)\right)_{1 \leq \mu \leq n ; 1 \leq i \leq k}$.

Hence we deduce that the set of (non algebraic) observable $(n-1)$-forms on $\mathcal{M}^{d D W}$ contains the set of $(n-1)$-forms $F$ on $\mathcal{M}^{d D W}$ such that, at each point $m \in \mathcal{M}^{d D W}, d F_{m}$ has the form

$$
\left.d F_{m}=\left(a^{\mu} \frac{\partial}{\partial x^{\mu}}+b^{i} \frac{\partial}{\partial y^{i}}\right)\right\lrcorner \Omega+\sum_{j=1}^{n} \sum_{i_{1}<\cdots<i_{j}} \sum_{\mu_{1}<\cdots<\mu_{j}} f_{i_{1} \cdots i_{p}}^{\mu_{1} \cdots \mu_{p}} \omega_{\mu_{1} \cdots \mu_{p}}^{i_{1} \cdots i_{p}} .
$$

Let us denote by $\mathfrak{P}_{0}^{n-1} \Lambda^{n} T^{*}(\mathcal{X} \times \mathcal{Y})_{\mid \mathcal{M}^{d D W}}$ this set. An equivalent definition could be that $\mathfrak{P}_{0}^{n-1} \Lambda^{n} T^{*}(\mathcal{X} \times \mathcal{Y})_{\mid \mathcal{M}^{d D W}}$ is the set of the restrictions of algebraic observable forms $\widetilde{F} \in \mathfrak{P}_{0}^{n-1} \Lambda^{n} T^{*}(\mathcal{X} \times \mathcal{Y})$ on $\mathcal{M}^{d D W}$ (and this is the reason for this notation). Hence $\mathfrak{P}^{n-1} \mathcal{M}^{d D W} \supset \mathfrak{P}_{0}^{n-1} \Lambda^{n} T^{*}(\mathcal{X} \times \mathcal{Y})_{\mid \mathcal{M}} d D W$. We will see in Section 5 that the reverse inclusion holds, so that actually $\mathfrak{P}^{n-1} \mathcal{M}^{d D W}=\mathfrak{P}_{0}^{n-1} \Lambda^{n} T^{*}(\mathcal{X} \times \mathcal{Y})_{\mid \mathcal{M}^{d D W}}$, with $\mathcal{O}_{m} \mathcal{M}^{d D W}=\{X \in$ $\left.D_{m}^{n} \mathcal{M}^{d D W} / \omega(X) \neq 0\right\}$.

\subsubsection{Invariance properties along pseudo-fibers}

In the following if $\zeta$ is a smooth vector field, we denote by $e^{s \zeta}$ (for $s \in I$, where $I$ is an interval of $\mathbb{R}$ ) its flow mapping. And if $E$ is any subset of $\mathcal{M}$, we denote by $E_{s}:=e^{s \zeta}(E)$ its image by $e^{s \zeta}$.

Lemma 4.2. Let $\Gamma \in \mathcal{E}^{\mathcal{H}}$ be a Hamiltonian $n$-curve and $\zeta$ be a vector field which is a smooth section of $L^{\mathcal{H}}$ (see Definition 2.4). Suppose that, for all $s \in I, \Gamma_{s}$ is a Hamiltonian $n$-curve $e^{4}$. Let $\Sigma$ be a smooth $(n-1)$-dimensional submanifold of $\Gamma$ and $F \in \mathfrak{P}_{0}^{n-1} \mathcal{M}$. If one of the two following hypotheses is satisfied: either

(a) $\partial \Sigma=\emptyset$, or

(b) $\zeta \_F=0$ everywhere, then

$$
\forall s \in I, \quad \int_{\Sigma} F=\int_{\Sigma_{s}} F
$$

\footnotetext{
${ }^{4}$ Observe that this hypothesis is true if $\mathcal{H}$ is pataplectic invariant, see Definition 2.5
} 
i.e., the integral of $F$ on the image of $\Sigma$ by $e^{s \zeta}$ does not depend on $s$.

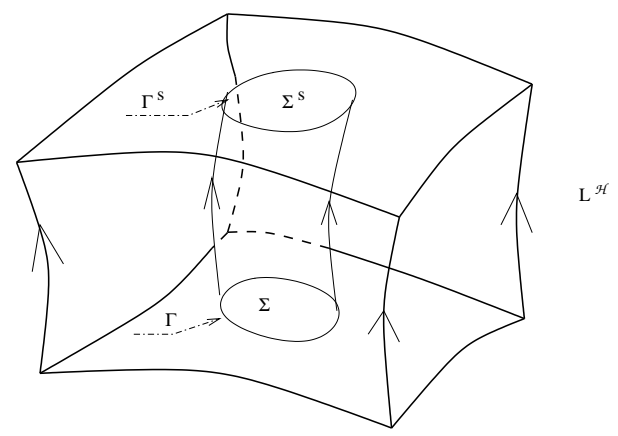

Figure 1: Invariance of an observable functional along the generalized pseudofiber directions as in Lemma 4.2.

Proof. Let us introduce some extra notations: $\sigma: I \times \Gamma \longrightarrow \mathcal{M}$ is the $\operatorname{map}(s, m) \longmapsto \sigma(s, m):=e^{s \zeta}(m)$. Moreover for all $m \in \Sigma_{s} \cup \partial \Sigma_{s}$ we consider a basis $\left(X_{1}, \ldots, X_{n}\right)$ of $T_{m} \Gamma_{s}$ such that $X:=X_{1} \wedge \cdots \wedge X_{n} \in[X]_{m}^{\mathcal{H}}$, $\left(X_{2}, \ldots, X_{n}\right)$ is a basis of $T_{m} \Sigma_{s}$ and, if $m \in \partial \Sigma_{s},\left(X_{3}, \ldots, X_{n}\right)$ is a basis of $T_{m} \partial \Sigma_{s}$. Lastly we let $\left(\theta^{1}, \ldots, \theta^{n}\right)$ be a basis of $T_{m}^{*} \Gamma_{s}$, dual of $\left(X_{1}, \ldots, X_{n}\right)$. We first note that

$$
\int_{(0, s) \times \partial \Sigma} \sigma^{*} F=0
$$

either because $\partial \Sigma=\emptyset$ (a) or because, if $\partial \Sigma \neq \emptyset$, this integral is equal to

$$
\int_{(0, s) \times \partial \Sigma} F_{\sigma(s, m)}\left(\zeta, X_{3}, \ldots, X_{n}\right) d s \wedge \theta^{3} \wedge \cdots \wedge \theta^{n}
$$

which vanishes by (b). Thus

$$
\begin{aligned}
\int_{\Sigma_{s}} F-\int_{\Sigma} F & =\int_{\Sigma}\left(e^{s \zeta}\right)^{*} F-F \\
& =\int_{\Sigma}\left(e^{s \zeta}\right)^{*} F-F-\int_{(0, s) \times \partial \Sigma} \sigma^{*} F \\
& =\int_{\partial((0, s) \times \Sigma)} \sigma^{*} F \\
& =\int_{(0, s) \times \Sigma} d\left(\sigma^{*} F\right) \\
& =\int_{(0, s) \times \Sigma} \sigma^{*} d F \\
& =\int_{\sigma((0, s) \times \Sigma)} d F_{\sigma(s, m)}\left(\zeta, X_{2}, \ldots, X_{n}\right) d s \wedge \theta^{2} \wedge \cdots \wedge \theta^{n} .
\end{aligned}
$$


But since $F \in \mathfrak{P}_{0}^{n-1} \mathcal{M}$, we have that

$$
\begin{aligned}
d F_{\sigma(s, m)}\left(\zeta, X_{2}, \ldots, X_{n}\right) & =-\Omega\left(\xi_{F}, \zeta, X_{2}, \ldots, X_{n}\right) \\
& =\Omega\left(\zeta, \xi_{F}, X_{2}, \ldots, X_{n}\right) \\
& \left.=\left\langle\xi_{F} \wedge X_{2} \wedge \cdots \wedge X_{n}, \zeta\right\lrcorner \Omega\right\rangle .
\end{aligned}
$$

Now the key observation is that $\xi_{F} \wedge X_{2} \wedge \cdots \wedge X_{n} \in T_{X} D_{m}^{n} \mathcal{M}$ and so the hypothesis of the Lemma implies that $\left.\left\langle\xi_{F} \wedge X_{2} \wedge \cdots \wedge X_{n}, \zeta\right\lrcorner \Omega\right\rangle=0$. Hence (12) is satisfied.

Example 6 (Algebraic observable $(n-1)$-forms satisfying the assumption (b) in Lemma 4.2). If $\mathcal{M}=\Lambda^{n} T^{*} \mathcal{N}$ and if $\mathcal{H}$ is a Legendre image Hamiltonian then any $(n-1)$-form $F$ of the type $F=\xi\lrcorner \theta$, where $\xi$ is a vector field on $\mathcal{N}$, is algebraic observable (see [14], [8]). But as pointed out in Section 2.2 (definition 2.4) since $L_{m}^{\mathcal{H}} \subset \operatorname{Ker} d \Pi_{m}$ any vector field $\zeta$ which is a section of $L^{\mathcal{H}}$ is necessarily "vertical" and satisfies $\left.\zeta\right\lrcorner \theta=0$. Hence $\zeta\lrcorner F=0$. Such (n-1)-forms $\xi\lrcorner \theta$ correspond to components of the momentum and the energy-momentum of the field (see [14]).

\subsubsection{Poisson brackets between observable $(n-1)$-forms}

There is a natural way to construct a Poisson bracket $\{\cdot, \cdot\}: \mathfrak{P}_{0}^{n-1} \mathcal{M} \times$ $\mathfrak{P}_{0}^{n-1} \mathcal{M} \longmapsto \mathfrak{P}_{0}^{n-1} \mathcal{M}$. To each algebraic observable forms $F, G \in \mathfrak{P}_{0}^{n-1} \mathcal{M}$ we associate first the vector fields $\xi_{F}$ and $\xi_{G}$ such that $\left.\xi_{F}\right\lrcorner \Omega+d F=$ $\left.\xi_{G}\right\lrcorner \Omega+d G=0$ and then the $(n-1)$-form

$$
\left.\{F, G\}:=\xi_{F} \wedge \xi_{G}\right\lrcorner \Omega .
$$

It can be shown (see [14]) that $\{F, G\} \in \mathfrak{P}_{0}^{n-1} \mathcal{M}$ and that

$$
\left.d\{F, G\}+\left[\xi_{F}, \xi_{G}\right]\right\lrcorner \Omega=0,
$$

where $[\cdot, \cdot]$ is the Lie bracket on vector fields. Moreover this bracket satisfies the Jacobi condition modulo an exact term ${ }^{5}$ (see [14])

$$
\left.\{\{F, G\}, H\}+\{\{G, H\}, F\}+\{\{H, F\}, G\}=d\left(\xi_{F} \wedge \xi_{G} \wedge \xi_{H}\right\lrcorner \Omega\right) .
$$

\footnotetext{
${ }^{5}$ Note that in case where the multisymplectic manifold $(\mathcal{M}, \Omega)$ is exact in the sense of M. Forger, C. Paufler and H. Römer [8], i.e., if there exists an $n$-form $\theta$ such that $\Omega=d \theta$ (beware that our sign conventions differ from [8]), an alternative Poisson bracket can be defined:

$$
\left.\left.\left.\{F, G\}_{\theta}:=\{F, G\}+d\left(\xi_{G}\right\lrcorner F-\xi_{F}\right\lrcorner G+\xi_{F} \wedge \xi_{G}\right\lrcorner \theta\right) .
$$

Then this bracket satisfies the Jacobi identity (in particular with a right hand side equal to 0$)$, see [7], [8].
} 
As an application of this definition, for any slice $\Sigma$ of codimension 1 we can define a Poisson bracket between the observable functionals $\int_{\Sigma} F$ and $\int_{\Sigma} G$ by $\forall \Gamma \in \mathcal{E}^{\mathcal{H}}$,

$$
\left\{\int_{\Sigma} F, \int_{\Sigma} G\right\}(\Gamma):=\int_{\Sigma \cap \Gamma}\{F, G\}
$$

If $\partial \Gamma=\emptyset$, it is clear that this Poisson bracket satisfies the Jacobi identity. Computations in [23], [21], [14] show that this Poisson bracket coincides with the Poisson bracket of the standard canonical formalism used for quantum field theory.

One can try to extend the bracket between forms in $\mathfrak{P}_{0}^{n-1} \mathcal{M}$ to forms in $\mathfrak{P}^{n-1} \mathcal{M}$ through different strategies:

- By exploiting the relation

$$
\left.\left.\{F, G\}=\xi_{F}\right\lrcorner d G=-\xi_{G}\right\lrcorner d F,
$$

which holds for all $F, G \in \mathfrak{P}_{0}^{n-1} \mathcal{M}$. A natural definition is to set:

$$
\left.\forall F \in \mathfrak{P}_{0}^{n-1} \mathcal{M}, \forall G \in \mathfrak{P}^{n-1} \mathcal{M}, \quad\{F, G\}=-\{G, F\}:=\xi_{F}\right\lrcorner d G .
$$

In [14] we call this operation an external Poisson bracket.

- If we know that there is an embedding $\iota: \mathcal{M} \longrightarrow \widehat{\mathcal{M}}$, into a higher dimensional pataplectic manifold $(\widehat{\mathcal{M}}, \widehat{\Omega})$, and that (i) $\mathfrak{P}_{0}^{n-1} \widehat{\mathcal{M}}=$ $\mathfrak{P}^{n-1} \widehat{\mathcal{M}}$, (ii) the pull-back mapping $\mathfrak{P}_{0}^{n-1} \widehat{\mathcal{M}} \longrightarrow \mathfrak{P}^{n-1} \mathcal{M}: \widehat{F} \longmapsto \iota^{*} \widehat{F}$ is - modulo the set of closed $(n-1)$-forms on $\widehat{\mathcal{M}}$ which vanish on $\mathcal{M}$ - an isomorphism. Then there exists a unique Poisson bracket on $\mathfrak{P}^{n-1} \mathcal{M}$ which is the image of the Poisson bracket on $\mathfrak{P}_{0}^{n-1} \widehat{\mathcal{M}}$.

This situation is achieved for instance if $\mathcal{M}$ is a submanifold of $\Lambda^{n} T^{*} \mathcal{N}$, a situation which arises after a Legendre transform. This will lead basically to the same structure as the external Poisson bracket. In more general cases the question of extending $\mathcal{M}$ into $\widehat{\mathcal{M}}$ is relatively subtle and is discussed in the paper [18].

\subsection{Algebraic observable $(p-1)$-forms}

It is worth asking about the relevant definition of algebraic observable $(p-1)$ forms. Indeed a first possibility is to generalize directly Definition 4.1: we would say that the $(p-1)$-form $F$ is algebraic observable if there exists a $(1+n-p)$-multivector field $\xi_{F}$ such that $\left.d F+\xi_{F}\right\lrcorner \Omega=0$. Note that in this case $\xi_{F}$ is not unique in general. This approach has been proposed 
by I. Kanatchikov in [21] and [22]. It allows us to define a nice notion of Poisson bracket between such forms and leads to a structure of graded Lie algebra. However this definition has not good dynamical properties and in particular there is no analogue of Theorem 3.1 for such forms. In other words our definition 3.3 of (non algebraic) observable $(p-1)$-forms results from the search for the more general hypothesis for Theorem 3.1 to be true, but $(p-1)$-forms which are observable according to Kanatchikov are not observable in the sense of our Definition 3.3. Here we prefer to privilege the dynamical properties instead trying to generalize Noether's theorem to $(p-1)$-forms.

\subsubsection{Definitions and basic properties}

We simply adapt Definitions 3.2, 3.3 and 3.4 by replacing $P^{n} T_{m}^{*} \mathcal{M}$ of Definition 3.1 by its subset $P_{0}^{n} T_{m}^{*} \mathcal{M}$ of Definition 4.1: it leads to the notions of algebraic copolarization $P_{0}^{*} T^{*} \mathcal{M}$, of the set $\mathfrak{P}_{0}^{p-1} \mathcal{M}$ of algebraic observable $(p-1)$-forms and of algebraic polarization $P_{0}^{*} T \mathcal{M}$. Of course in the case of a pataplectic manifold algebraic and non algebraic notions coincide.

A consequence of these definitions is that for any $1 \leq p \leq n$, for any algebraic observable $(p-1)$-forms $F \in \mathfrak{P}_{0}^{p-1} \mathcal{M}$ and for any $\phi \in P_{0}^{n-p} T_{m}^{\star} \mathcal{M}$, there exists a unique vector $\xi_{F}(\phi) \in T_{m} \mathcal{M}$ such that $\left.\phi \wedge d F+\xi_{F}(\phi)\right\lrcorner \Omega=0$. We thus obtain a linear mapping

$$
\begin{aligned}
\xi_{F}: P_{0}^{n-p} T_{m}^{\star} \mathcal{M} & \longrightarrow T_{m} \mathcal{M} \\
\phi & \longmapsto \xi_{F}(\phi) .
\end{aligned}
$$

Hence we can associate to $F$ the tensor field $\xi_{F}$. By duality between $P_{0}^{n-p} T_{m}^{\star} \mathcal{M}$ and $P_{0}^{n-p} T_{m} \mathcal{M}, \xi_{F}$ can also be identified with a section of the bundle $P_{0}^{n-p} T \mathcal{M} \otimes_{\mathcal{M}} T \mathcal{M}$.

Moreover an alternative definition of the pseudobracket (see Definition $3.5)$ can be given using the tensor field $\xi_{F}$ defined by (13).

Lemma 4.3. For any Hamiltonian function $\mathcal{H}$ and any $F \in \mathfrak{P}_{0}^{p-1} \mathcal{M}$, we have

$$
\left.\{\mathcal{H}, F\}=-\xi_{F}\right\lrcorner d \mathcal{H}
$$

where the right hand side is the section of $P_{0}^{n-p} T \mathcal{M}$ defined by

$$
\left.\left.\left\langle\xi_{F}\right\lrcorner d \mathcal{H}, \phi\right\rangle:=\xi_{F}(\phi)\right\lrcorner d \mathcal{H}, \quad \forall \phi \in P_{0}^{n-p} T^{\star} \mathcal{M} .
$$


Proof. Starting from Definition 3.5, we have $\forall \phi \in P_{0}^{n-p} T^{\star} \mathcal{M}$,

$$
\begin{aligned}
\langle\{\mathcal{H}, F\}, \phi\rangle & =(-1)^{(n-p) p}\left\langle[X]^{\mathcal{H}}\llcorner d F, \phi\rangle\right. \\
& =(-1)^{(n-p) p}\left\langle[X]^{\mathcal{H}}, d F \wedge \phi\right\rangle \\
& =\left\langle[X]^{\mathcal{H}}, \phi \wedge d F\right\rangle \\
& \left.=-\left\langle[X]^{\mathcal{H}}, \xi_{F}(\phi)\right\lrcorner \Omega\right\rangle \\
& \left.\left.=-(-1)^{n} \xi_{F}(\phi)\right\lrcorner[X]^{\mathcal{H}}\right\lrcorner \Omega \\
& \left.=-\xi_{F}(\phi)\right\lrcorner d \mathcal{H} .
\end{aligned}
$$

The price we have to pay is that the notion of Poisson bracket between $(p-1)$-forms is now a much more delicate task than in the framework of Kanatchikov. This question is the subject of the next paragraph.

\subsubsection{Brackets between algebraic observable $(p-1)$-forms}

We now consider algebraic observable $(p-1)$-forms for $1 \leq p \leq n$ and discuss the possibility of defining a Poisson bracket between these observable forms, which could be relevant for quantization. This is slightly more delicate than for forms of degree $n-1$ and the definitions proposed here are based on empirical observations. We first assume a further hypothesis on the copolarization (which is satisfied on $\Lambda^{n} T^{*} \mathcal{N}$ ).

We first recall a definition which was given in [16]: given any $X=X_{1} \wedge$ $\cdots \wedge X_{n} \in D_{m}^{n} \mathcal{M}$ and any form $a \in T_{m}^{*} \mathcal{M}$ we will write that $a_{\mid X} \neq 0$ if and only if $\left(a\left(X_{1}\right), \ldots, a\left(X_{n}\right)\right) \neq 0$. We will say that a function $f \in \mathcal{C}^{1}(\mathcal{M}, \mathbb{R})$ is 1-regular if and only if we have

$$
\forall m \in \mathcal{M}, \forall X \in[X]_{m}^{\mathcal{H}}, \quad d f_{m \mid X} \neq 0
$$

Hypothesis on $\mathfrak{P}_{0}^{1} \mathcal{M}$. We suppose that any 1-regular function $f \in$ $\mathcal{C}^{1}(\mathcal{M}, \mathbb{R})$ satisfies $^{6}$

(i) $f \in \mathfrak{P}_{0}^{1} \mathcal{M}$

(ii) For all infinitesimal symplectomorphism $\left.\xi \in \mathfrak{s p}_{0} \mathcal{M}, \xi\right\lrcorner d f=0$.

\footnotetext{
${ }^{6}$ These assumptions are actually satisfied in $\mathfrak{P}^{n-1} \mathcal{M}$.
} 
Now let $1 \leq p, q \leq n$ and $F \in \mathfrak{P}_{0}^{p-1} \mathcal{M}$ and $G \in \mathfrak{P}_{0}^{q-1} \mathcal{M}$ and let us analyze what condition should satisfy the bracket $\{F, G\}$. We will consider smooth functions $f^{1}, \ldots, f^{n-p}, g^{1}, \ldots, g^{n-q}$ and $t$ on $\mathcal{M}$. We assume that all these functions are 1-regular and that $d f_{m}^{1} \wedge \cdots \wedge d f_{m}^{n-p} \wedge d g_{m}^{1} \wedge \cdots \wedge d g_{m}^{n-q} \neq 0$. Then, because of hypothesis (i),

$$
\widetilde{F}:=d f^{1} \wedge \cdots \wedge d f^{n-p} \wedge F, \quad \widetilde{G}:=d g^{1} \wedge \cdots \wedge d g^{n-q} \wedge G \in \mathfrak{P}_{0}^{n-1} \mathcal{M} .
$$

Lastly let $\Gamma$ be a Hamiltonian $n$-curve and $\Sigma$ be a level set of $t$. Then

$$
\left\{\int_{\Sigma} \widetilde{F}, \int_{\Sigma} \widetilde{G}\right\}(\Gamma)=\left(\int_{\Sigma}\{\widetilde{F}, \widetilde{G}\}\right)(\Gamma)=\int_{\Sigma \cap \Gamma}\{\widetilde{F}, \widetilde{G}\}
$$

We now suppose that the functions $f:=\left(f^{1}, \ldots, f^{n-p}\right)$ and $g:=\left(g^{1}, \ldots\right.$, $\left.g^{n-q}\right)$ concentrate around submanifolds denoted respectively by $\widehat{\gamma}_{f}$ and $\widehat{\gamma}_{g}$ of codimension $n-p$ and $n-q$ respectively. More precisely we suppose that $d f^{1} \wedge \cdots \wedge d f^{n-p}$ (resp. $d g^{1} \wedge \cdots \wedge d g^{n-q}$ ) is zero outside a tubular neighborhood of $\widehat{\gamma}_{f}$ (resp. of $\widehat{\gamma}_{g}$ ) of width $\varepsilon$ and that the integral of $d f^{1} \wedge \cdots \wedge d f^{n-p}$ (resp. $\left.d g^{1} \wedge \cdots \wedge d g^{n-q}\right)$ on a disc submanifold of dimension $n-p($ resp. $n-q)$ which cuts transversally $\widehat{\gamma}_{f}$ (resp. $\widehat{\gamma}_{g}$ ) is equal to 1 . Moreover we suppose that $\widehat{\gamma}_{f}$ and $\widehat{\gamma}_{g}$ cut transversally $\Sigma \cap \Gamma$ along submanifolds denoted by $\gamma_{f}$ and $\gamma_{g}$ respectively. Then, as $\varepsilon \rightarrow 0$, we have

$$
\begin{gathered}
\int_{\Sigma \cap \Gamma} d f^{1} \wedge \cdots \wedge d f^{n-p} \wedge F \rightarrow \int_{\Sigma \cap \widehat{\gamma}_{f} \cap \Gamma} F \\
\int_{\Sigma \cap \Gamma} d g^{1} \wedge \cdots \wedge d g^{n-q} \wedge G \rightarrow \int_{\Sigma \cap \widehat{\gamma}_{g} \cap \Gamma} G .
\end{gathered}
$$

This tells us that the left hand side of (17) is an approximation for

$$
\left\{\int_{\Sigma \cap \widehat{\gamma}_{f}} F, \int_{\Sigma \cap \widehat{\gamma}_{g}} G\right\}(\Gamma) .
$$

We now want to compute what is the limit of the right hand side of (17). Using the hypothesis (ii) we have

$$
\begin{aligned}
\{\widetilde{F}, \widetilde{G}\} & \left.=\xi_{\widetilde{F}}\right\lrcorner d\left(d g^{1} \wedge \cdots \wedge d g^{n-q} \wedge G\right) \\
& \left.=(-1)^{n-q} \xi_{\widetilde{F}}\right\lrcorner d g^{1} \wedge \cdots \wedge d g^{n-q} \wedge d G \\
& \left.=d g^{1} \wedge \cdots \wedge d g^{n-q} \wedge\left(\xi_{\widetilde{F}}\right\lrcorner d G\right) .
\end{aligned}
$$

And we have similarly:

$$
\left.\{\widetilde{F}, \widetilde{G}\}=-d f^{1} \wedge \cdots \wedge d f^{n-p} \wedge\left(\xi_{\widetilde{G}}\right\lrcorner d F\right) .
$$

We now use the following result. 


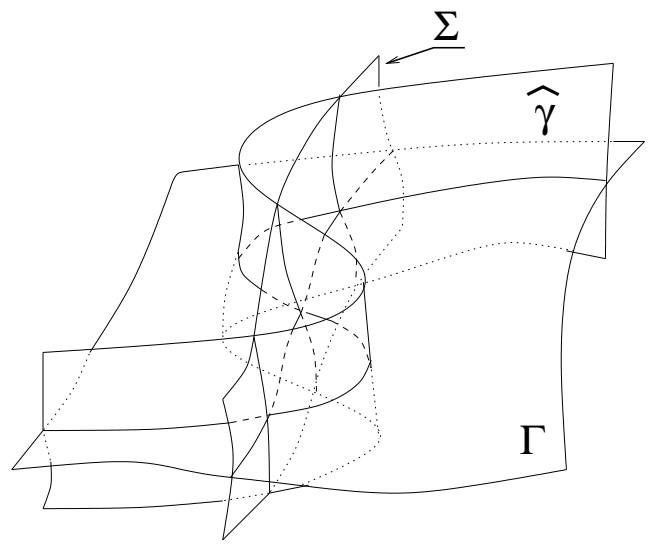

Figure 2: Intersection of $\Gamma, \widehat{\gamma}$ and $\Sigma$.

Lemma 4.4. Let $\phi \in \Lambda^{n-1} T_{m}^{*} \mathcal{M}$ with $\phi \neq 0$ and $1 \leq p, q \leq n$ such that $p+q \geq n+1$. Suppose that there exists $2 n-p-q$ linearly independent 1 forms $a^{1}, \ldots, a^{n-p}, b^{1}, \ldots, b^{n-q} \in T_{m}^{*} \mathcal{M}, \alpha \in \Lambda^{p-1} T_{m}^{*} \mathcal{M}$ and $\beta \in \Lambda^{q-1} T_{m}^{*} \mathcal{M}$ such that $\phi=a^{1} \wedge \cdots \wedge a^{n-p} \wedge \alpha$ and $\phi=b^{1} \wedge \cdots \wedge b^{n-q} \wedge \beta$. Then there exists $\chi \in \Lambda^{p+q-n-1} T_{m}^{*} \mathcal{M}$ such that $\phi=a^{1} \wedge \cdots \wedge a^{n-p} \wedge b^{1} \wedge \cdots \wedge b^{n-q} \wedge \chi$. This $\chi$ is not unique in general and is defined modulo forms in the ideal in $\Lambda^{*} T_{m}^{*} \mathcal{M}$ spanned by the $a_{j}$ 's and the $b_{j}$ 's. However it is a unique real scalar if $p+q=n+1$.

Proof. This is a consequence of Proposition 1.4 in [2]. The idea is based on the observation that $a^{1}, \ldots, a^{n-p}, b^{1}, \ldots, b^{n-q}$ are in $\left\{a \in T_{m}^{*} \mathcal{M} / a \wedge \phi=\right.$ $0\}$.

We deduce from Lemma 4.4 that there exist a form $\chi \in \Lambda^{p+q-n-1} T_{m}^{*} \mathcal{M}$ (not unique a priori) such that $\{\widetilde{F}, \widetilde{G}\}=d f^{1} \wedge \cdots \wedge d f^{n-p} \wedge d g^{1} \wedge \cdots \wedge d g^{n-q} \wedge \chi$. We thus require that

$$
\{\widetilde{F}, \widetilde{G}\}=d f^{1} \wedge \cdots \wedge d f^{n-p} \wedge d g^{1} \wedge \cdots \wedge d g^{n-q} \wedge\{F, G\} .
$$

This does not characterize completely $\{F, G\}$, unless $p+q=n+1$, the case $\{F, G\}$ where is a scalar. We can now write the right hand side of (17) as

$$
\int_{\Sigma \cap \Gamma} d f^{1} \wedge \cdots \wedge d f^{n-p} \wedge d g^{1} \wedge \cdots \wedge d g^{n-q} \wedge\{F, G\}
$$

Letting $\varepsilon \rightarrow 0$, and assuming that $\widehat{\gamma}_{f}$ and $\widehat{\gamma}_{g}$ cross transversally this integral converges to

$$
\int_{\Sigma \cap \widehat{\gamma}_{f} \cap \widehat{\gamma}_{g} \cap \Gamma}\{F, G\},
$$


so that we have

$$
\left\{\int_{\Sigma \cap \widehat{\gamma}_{f}} F, \int_{\Sigma \cap \widehat{\gamma}_{g}} G\right\}(\Gamma)=\int_{\Sigma \cap \widehat{\gamma}_{f} \cap \widehat{\gamma}_{g} \cap \Gamma}\{F, G\} .
$$

Here the intersection $\Sigma \cap \widehat{\gamma}_{f} \cap \widehat{\gamma}_{g} \cap \Gamma$ is oriented by assuming that $X\llcorner d t \wedge$ $d f \wedge d g$ is oriented positively, if $X \in[X]^{\mathcal{H}}$ orients positively $T_{m} \Gamma$. Hence if we had started with $\int_{\Sigma \cap \Gamma}\{\widetilde{F}, \widetilde{G}\}=-\int_{\Sigma \cap \Gamma}\{\widetilde{G}, \widetilde{F}\}$ we would have obtained $-\int_{\Sigma \cap \widehat{\gamma}_{g} \cap \widehat{\gamma}_{f} \cap \Gamma}\{G, F\}=-(-1)^{(n-p)(n-q)} \int_{\Sigma \cap \widehat{\gamma}_{f} \cap \widehat{\gamma}_{g} \cap \Gamma}\{G, F\}$. Since the resulting brackets should coincide we deduce that

$$
\{F, G\}+(-1)^{(n-p)(n-q)}\{G, F\}=0 .
$$

Conclusion. In two cases we can guess a more direct definition of $\{F, G\}$. First when one of the two forms $F$ or $G$ is in $\mathfrak{P}_{0}^{n-1} \mathcal{M}$, let us say $F \in \mathfrak{P}_{0}^{p-1} \mathcal{M}$ and $G \in \mathfrak{P}_{0}^{n-1} \mathcal{M}$ : then we let

$$
\left.\{F, G\}:=-\xi_{G}\right\lrcorner d F .
$$

This is the idea of external bracket as in Paragraph 4.1.4. We remark that if $f^{1}, \ldots, f^{n-p}$ are in $\mathfrak{P}_{0}^{1} \mathcal{M}$ and are such that $\left.\xi_{G}\right\lrcorner d f^{1} \wedge \cdots \wedge d f^{n-p}=0$, then $d f^{1} \wedge \cdots \wedge d f^{n-p} \wedge F \in \mathfrak{P}_{0}^{n-1} \mathcal{M}$ and

$$
\left\{d f^{1} \wedge \cdots \wedge d f^{n-p} \wedge F, G\right\}=d f^{1} \wedge \cdots \wedge d f^{n-p} \wedge\{F, G\}
$$

so that the requirement (18) is satisfied.

Second if $F \in \mathfrak{P}_{0}^{p-1} \mathcal{M}, G \in \mathfrak{P}_{0}^{q-1} \mathcal{M}$, where $1<p, q<n$ and $p+q=n+1$ : then $\{F, G\}$ is just a scalar and so is characterized by (18).

Example 7 (Sigma models). Let $\mathcal{M}:=\Lambda^{n} T^{\star}(\mathcal{X} \times \mathcal{Y})$ as in Section 2 . For simplicity we restrict ourself to the de Donder-Weyl submanifold $\mathcal{M}^{d D W}$ (see Section 2.3), so that the Poincaré-Cartan form is $\theta=e \omega+p_{i}^{\mu} d y^{i} \wedge \omega_{\mu}$ and the multisymplectic form is $\Omega=d \theta$. Let $\phi$ be a function on $\mathcal{X}$ and consider the observable 0 -form $y^{i}$ (for $1 \leq i \leq k$ ) and the observable $(n-1)$-form $\left.P_{j, \phi}:=\phi(x) \partial / \partial y^{j}\right\lrcorner \theta$. Then $\xi_{P_{j, \phi}}=\phi \partial / \partial y^{j}-p_{j}^{\mu}\left(\partial \phi / \partial x^{\mu}\right) \partial / \partial e$ and thus $\left.\left\{P_{j, \phi}, y^{i}\right\}=\xi_{P_{j, \phi}}\right\lrcorner d y^{i}=\delta_{j}^{i} \phi$. It gives the following bracket for observable functionals

$$
\left\{\int_{\Sigma} P_{j, \phi}, \int_{\Sigma \cap \widehat{\gamma}} y^{i}\right\}(\Gamma)=\int_{\Sigma \cap \widehat{\gamma} \cap \Gamma} \delta_{j}^{i} \phi(x)=\delta_{j}^{i} \sum_{m \in \Sigma \cap \widehat{\gamma} \cap \Gamma} \operatorname{sign}(m) \phi(m),
$$

where $\Sigma, \widehat{\gamma}$ and $\Gamma$ are supposed to cross transversally and $\operatorname{sign}(m)$ accounts for the orientation of their intersection points. 
Example $4^{\prime \prime}$ (Maxwell equations). In this case we find that, for all functions $f, g_{1}, g_{2}: \mathcal{M}^{\mathrm{Max}} \longrightarrow \mathbb{R}$ whose differentials are proper on $\mathcal{O}_{m}$, $\left\{d f \wedge \pi, d g_{1} \wedge d g_{2} \wedge a\right\}=d f \wedge d g_{1} \wedge d g_{2}$. We hence deduce that $\{\pi, a\}=1$ : these forms are canonically conjugate. We deduce the following bracket for observable functionals

$$
\left\{\int_{\Sigma \cap \widehat{\gamma}_{f}} \pi, \int_{\Sigma \cap \widehat{\gamma}_{g}} a\right\}(\Gamma)=\sum_{m \in \Sigma \cap \widehat{\gamma}_{f} \cap \widehat{\gamma}_{g} \cap \Gamma} \operatorname{sign}(m),
$$

where $\Sigma \cap \widehat{\gamma}_{f} \cap \Gamma$ is a surface and $\Sigma \cap \widehat{\gamma}_{g} \cap \Gamma$ is a curve in the three-dimensional space $\Sigma \cap \Gamma$. Note that this conclusion was achieved by I. Kanatchikov with its definition of bracket $\left.\{\pi, a\}_{\text {Kana }}:=\xi_{\pi}\right\lrcorner d a$, where $\xi_{\pi} \in \Lambda^{2} T_{m}^{*} \mathcal{M}^{\text {Max }}$ is such that $\left.\xi_{\pi}\right\lrcorner \Omega=d \pi$. But there by choosing $\xi_{\pi}=(1 / 2) \sum_{\mu}\left(\partial / \partial a_{\mu}\right) \wedge\left(\partial / \partial x^{\mu}\right)$ one finds (in our convention) that $\{\pi, a\}_{\text {Kana }}=n / 2(=2$, if $n=4)$. So the two brackets differ (the new bracket in this paper differs also from the one that we proposed in [14]).

\section{The case of $\Lambda^{n} T^{\star} \mathcal{N}$}

We will here study algebraic and non algebraic observable $(n-1)$-forms in $\Lambda^{n} T^{\star} \mathcal{N}$ and prove in particular that $\Lambda^{n} T^{\star} \mathcal{N}$ is pataplectic. This part can be seen as a complement of an analysis of other properties of $\Lambda^{n} T^{\star} \mathcal{N}$ given in [16]. In particular we use the same notations: since we are interested here in local properties of $\mathcal{M}$, we will use local coordinates $m=(q, p)=\left(q^{\alpha}, p_{\alpha_{1} \cdots \alpha_{n}}\right)$ on $\mathcal{M}$, and the multisymplectic form reads $\Omega=\sum_{\alpha_{1}<\cdots<\alpha_{n}} d p_{\alpha_{1} \cdots \alpha_{n}} \wedge d q^{\alpha_{1}} \wedge$ $\cdots \wedge d q^{\alpha_{n}}$. For $m=(q, p)$, we write

$$
d_{q} \mathcal{H}:=\sum_{1 \leq \alpha \leq n+k} \frac{\partial \mathcal{H}}{\partial q^{\alpha}} d q^{\alpha}, \quad d_{p} \mathcal{H}:=\sum_{1 \leq \alpha_{1}<\cdots<\alpha_{n} \leq n+k} \frac{\partial \mathcal{H}}{\partial p_{\alpha_{1} \cdots \alpha_{n}}} d p_{\alpha_{1} \cdots \alpha_{n}}
$$

so that $d \mathcal{H}=d_{q} \mathcal{H}+d_{p} \mathcal{H}$.

\subsection{Algebraic and non algebraic observable $(n-1)$-forms co- incide}

We show here that $\left(\Lambda^{n} T^{\star} \mathcal{N}, \Omega\right)$ is a pataplectic manifold.

Theorem 5.1. If $\mathcal{M}$ is an open subset of $\Lambda^{n} T^{\star} \mathcal{N}$, then $\mathfrak{P}_{0}^{n-1} \mathcal{M}=\mathfrak{P}^{n-1} \mathcal{M}$.

Proof. We already know that $\mathfrak{P}_{0}^{n-1} \mathcal{M} \subset \mathfrak{P}^{n-1} \mathcal{M}$. Hence we need to prove the reverse inclusion. So in the following we consider some $m \in \mathcal{M}$ and a 
form $a \in P_{m}^{n} \mathcal{M}$ and we will prove that there exists a vector field $\xi$ on $\mathcal{M}$ such that $a=\xi\lrcorner \Omega$. We write $\mathcal{O}_{m} \mathcal{M}:=\mathcal{O}_{m}^{a} \mathcal{M}$.

Step 1. We show that given $m=(q, p) \in \mathcal{M}$ it is possible to find $n+k$ vectors $\left(\widetilde{Q}_{1}, \ldots, \widetilde{Q}_{n+k}\right)$ of $T_{m} \mathcal{M}$ such that, if $\Pi_{*}\left(\widetilde{Q}_{\alpha}\right)=: Q_{\alpha}$ (the image of $\widetilde{Q}_{\alpha}$ by the map $\left.\Pi: \mathcal{M} \longrightarrow \mathcal{N}\right)$, then $\left(Q_{1}, \ldots, Q_{n+k}\right)$ is a basis of $T_{q} \mathcal{N}$ and $\forall\left(\alpha_{1}, \ldots, \alpha_{n}\right)$ s.t. $1 \leq \alpha_{1}<\cdots<\alpha_{n} \leq n+k, \widetilde{Q}_{\alpha_{1}} \wedge \cdots \wedge \widetilde{Q}_{\alpha_{n}} \in \mathcal{O}_{m} \mathcal{M}$.

This can be done by induction by using the fact that $\mathcal{O}_{m} \mathcal{M}$ is dense in $D_{m}^{n} \mathcal{M}$. We start from any family of vectors $\left(\widetilde{Q}_{1}^{0}, \ldots, \widetilde{Q}_{n+k}^{0}\right)$ of $T_{m} \mathcal{M}$ such that $\left(Q_{1}^{0}, \ldots, Q_{n+k}^{0}\right)$ is a basis of $T_{q} \mathcal{N}$ (where $\left.Q_{\alpha}^{0}:=\Pi_{*}\left(\widetilde{Q}_{\alpha}^{0}\right)\right)$. We then order the $\frac{(n+k) !}{n ! k !}$ multi-indices $\left(\alpha_{1}, \ldots, \alpha_{n}\right)$ such that $1 \leq \alpha_{1}<\cdots<\alpha_{n} \leq n+k$ (using for instance the dictionary rule). Using the density of $\mathcal{O}_{m} \mathcal{M}$ we can perturb slightly $\left(\widetilde{Q}_{1}^{0}, \ldots, \widetilde{Q}_{n+k}^{0}\right)$ into $\left(\widetilde{Q}_{1}^{1}, \ldots, \widetilde{Q}_{n+k}^{1}\right)$ in such a way that for instance $\widetilde{Q}_{1}^{1} \wedge \cdots \wedge \widetilde{Q}_{n}^{1} \in \mathcal{O}_{m} \mathcal{M}$ (assuming that $(1, \ldots, n)$ is the smallest index). Then we perturb further $\left(\widetilde{Q}_{1}^{1}, \ldots, \widetilde{Q}_{n+k}^{1}\right)$ into $\left(\widetilde{Q}_{1}^{2}, \ldots, \widetilde{Q}_{n+k}^{2}\right)$ in such a way that $\widetilde{Q}_{1}^{2} \wedge \cdots \wedge \widetilde{Q}_{n-1}^{2} \wedge \widetilde{Q}_{n+1}^{2} \in \mathcal{O}_{m} \mathcal{M}$ (assuming that $(1, \ldots, n-1, n+1$ ) is the next one). Using the fact that $\mathcal{O}_{m} \mathcal{M}$ is open we can do it in such a way that we still have $\widetilde{Q}_{1}^{2} \wedge \cdots \wedge \widetilde{Q}_{n}^{2} \in \mathcal{O}_{m} \mathcal{M}$. We proceed further until the conclusion is reached.

In the following we choose local coordinates around $m$ in such a way that $\widetilde{Q}_{\alpha}=\partial_{\alpha}+\sum_{1 \leq \alpha_{1}<\cdots<\alpha_{n} \leq n+k} P_{\alpha, \alpha_{1} \cdots \alpha_{n}} \partial^{\alpha_{1} \cdots \alpha_{n}}$.

Step 2. We choose a multi-index $\left(\alpha_{1}, \ldots, \alpha_{n}\right)$ with $1 \leq \alpha_{1}<\cdots<\alpha_{n} \leq n+k$ and define the set $\mathcal{O}_{m}^{\alpha_{1} \cdots \alpha_{n}} \mathcal{M}:=\mathcal{O}_{m} \mathcal{M} \cap D_{m}^{\alpha_{1} \cdots \alpha_{n}} \mathcal{M}$, where

$$
\begin{aligned}
D_{m}^{\alpha_{1} \cdots \alpha_{n}} \mathcal{M}:=\left\{X_{1} \wedge \cdots\right. & \wedge X_{n} \in D_{m}^{n} \mathcal{M} / \forall \mu, X_{\mu} \\
& \left.=\frac{\partial}{\partial q^{\alpha_{\mu}}}+\sum_{1 \leq \beta_{1}<\cdots<\beta_{n} \leq n+k} X_{\mu, \beta_{1} \cdots \beta_{n}} \frac{\partial}{\partial p^{\beta_{1} \cdots \beta_{n}}}\right\} .
\end{aligned}
$$

We want to understand the consequences of the relation

$$
\left.\left.\forall X, \tilde{X} \in \mathcal{O}_{m}^{\alpha_{1} \cdots \alpha_{n}} \mathcal{M}, \quad X\right\lrcorner \Omega=\tilde{X}\right\lrcorner \Omega \quad \Longrightarrow \quad a(X)=a(\tilde{X}) .
$$

Note that $\mathcal{O}_{m}^{\alpha_{1} \cdots \alpha_{n}} \mathcal{M}$ is open and non empty (since by the previous step, $\left.\widetilde{Q}_{\alpha_{1}} \wedge \cdots \wedge \widetilde{Q}_{\alpha_{n}} \in \mathcal{O}_{m}^{\alpha_{1} \cdots \alpha_{n}} \mathcal{M}\right)$. We also observe that, on $D_{m}^{\alpha_{1} \cdots \alpha_{n}} \mathcal{M}, X \longmapsto$ $X\lrcorner \Omega$ and $X \longmapsto a(X)$ are respectively an affine function and a polynomial function of the coordinates variables $X_{\mu, \beta_{1} \cdots \beta_{n}}$. Thus the following result implies that actually $\mathcal{O}_{m}^{\alpha_{1} \cdots \alpha_{n}} \mathcal{M}=D_{m}^{\alpha_{1} \cdots \alpha_{n}} \mathcal{M}$. 
Lemma 5.1. Let $N \in \mathbb{N}$ and let $P$ be a polynomial on $\mathbb{R}^{N}$ and $f_{1}, \ldots, f_{p}$ be affine functions on $\mathbb{R}^{N}$. Assume that there exists some $x_{0} \in \mathbb{R}^{N}$ and a neighborhood $V_{0}$ of $x_{0}$ in $\mathbb{R}^{N}$ such that

$$
\forall x, \widetilde{x} \in V_{0}, \quad \text { if } \forall j=1, \ldots, p, f_{j}(x)=f_{j}(\widetilde{x}), \quad \text { then } P(x)=P(\widetilde{x}) .
$$

Then this property is true on $\mathbb{R}^{N}$.

Proof. We can assume without loss of generality that the functions $f_{j}$ are linear and also choose coordinates on $\mathbb{R}^{N}$ such that $f_{j}(x)=x^{j}, \forall j=1, \ldots, p$. Then the assumption means that, on $V_{0}, P$ does not depend on $x^{p+1}, \ldots, x^{N}$. Since $P$ is a polynomial we deduce that $P$ is a polynomial on the variables $x^{1}, \ldots, x^{p}$ and so the property is true everywhere.

Step 3. Without loss of generality we will also assume in the following that $\left(\alpha_{1}, \ldots, \alpha_{n}\right)=(1, \ldots, n)$ for simplicity. We shall denote by $m^{I}$ all coordinates $q^{\alpha}$ and $p_{\alpha_{1} \cdots \alpha_{n}}$, so that we can write

$$
a=\sum_{I_{1}<\cdots<I_{n}} A_{I_{1} \cdots I_{n}} d m^{I_{1}} \wedge \cdots \wedge d m^{I_{n}}
$$

We will prove that if $\left(I_{1}, \ldots, I_{n}\right)$ is a multi-index such that

- $\left\{m^{I_{1}}, \ldots, m^{I_{n}}\right\}$ contains at least two distinct coordinates of the type $p_{\alpha_{1} \cdots \alpha_{n}}$ and

- $\left\{m^{I_{1}}, \ldots, m^{I_{n}}\right\}$ does not contain any $q^{\alpha}$, for $n+1 \leq \alpha \leq n+k$

then $A_{I_{1} \cdots I_{n}}=0$. Without loss of generality we can suppose that $\exists p \in \mathbb{N}$ such that $1 \leq p \leq n-2$ and

$$
\begin{aligned}
& m^{I_{1}}=q^{1}, \ldots, m^{I_{p}}=q^{p} \quad \text { and } \\
& m^{I_{p+1}}, \ldots, m^{I_{n}} \in\left\{p_{\alpha_{1} \cdots \alpha_{n}} / 1 \leq \alpha_{1}<\cdots<\alpha_{n} \leq n+k\right\} .
\end{aligned}
$$

We test property (20) specialized to the case where $X=X_{1} \wedge \cdots \wedge X_{n}$ with

$$
X_{\mu}=\frac{\partial}{\partial q^{\mu}}+\sum_{j=p+1}^{n} X_{\mu}^{I_{j}} \frac{\partial}{\partial m^{I_{j}}}, \quad \forall \mu=1, \ldots, n .
$$


Then

$$
\begin{array}{c|cccccc}
1 & \cdots & 0 & 0 & \cdots & 0 \\
\vdots & \ddots & \vdots & \vdots & & \vdots \\
0 & \cdots & 1 & 0 & \cdots & 0 \\
X_{1}^{I_{p+1}} & \cdots & X_{p}^{I_{p+1}} & X_{p+1}^{I_{p+1}} & \cdots & X_{n}^{I_{p+1}} \\
\vdots & & \vdots & \vdots & & \vdots \\
X_{1}^{I_{n}} & \cdots & X_{p}^{I_{n}} & X_{p+1}^{I_{n}} & \cdots & X_{n}^{I_{n}}
\end{array} \mid
$$

Note that we can write any $X \in D_{m}^{1 \cdots n} \mathcal{M}$ as $X=X_{1} \wedge \cdots \wedge X_{n}$ with

$$
X_{\mu}=\frac{\partial}{\partial q^{\mu}}+E_{\mu} \frac{\partial}{\partial p_{1 \cdots n}}+\sum_{\beta=n+1}^{n+k} M_{\mu, \beta}+R_{\mu}
$$

where

$$
\begin{aligned}
M_{\mu, \beta} & :=\sum_{\nu=1}^{n}(-1)^{n+\nu} M_{\mu, \beta}^{\nu} \partial^{1 \cdots \widehat{\nu} \cdots n \beta} \quad \text { and } \\
R_{\mu} & :=\sum_{\left(\alpha_{1}, \ldots, \alpha_{n}\right) \in I^{* *}} X_{\mu, \alpha_{1} \cdots \alpha_{n}} \partial^{\alpha_{1} \cdots \alpha_{n}}
\end{aligned}
$$

And then

$$
\left.(-1)^{n} X\right\lrcorner \Omega=d p_{1 \cdots n}-\sum_{\mu=1}^{n} E_{\mu} d q^{\mu}-\sum_{\beta=n+1}^{n+k}\left(\sum_{\mu=1}^{n} M_{\mu, \beta}^{\mu}\right) d q^{\beta} .
$$

Within our specialization this leads to the following key observation ${ }^{7}$ : at most one line $\left(X_{1}^{I_{j}}, \ldots, X_{n}^{I_{j}}\right)($ for $p+1 \leq j \leq n)$ in the $n \times n$ determinant in (21) is a function of $X\lrcorner \Omega$ (for $m^{I_{j}}=p_{1 \cdots n}$ ). In all other lines number $\nu$, where $p+1 \leq \nu \leq n$ and $\nu \neq j$, there is at most one component $X_{\mu}^{I_{\nu}}$ which is a function of $X\lrcorner \Omega$. All the other components are independent of $X\lrcorner \Omega$. Thus we have the following alternative.

(i) $\left\{m^{I_{p+1}}, \ldots, m^{I_{n}}\right\}$ does not contain $p_{1 \cdots n}$ (i.e., the line $\left(E_{1}, \ldots, E_{n}\right)$ does not appear in the $n \times n$ determinant in (21)), or

\footnotetext{
${ }^{7}$ Remark that each of the $n-p$ last lines in the $n \times n$ determinant in (21) is either $\left(E_{1}, \ldots, E_{n}\right)$ or of the type $\left(M_{1, \beta}^{\nu}, \cdots, M_{m, \beta}^{\nu}\right)$ or $\left(X_{1, \alpha_{1} \cdots \alpha_{n}}, \ldots, X_{n, \alpha_{1} \cdots \alpha_{n}}\right)$, for $\left(\alpha_{1}, \ldots, \alpha_{n}\right) \in I^{* *}$.
} 
(ii) $\left\{m^{I_{p+1}}, \ldots, m^{I_{n}}\right\}$ contains $p_{1 \cdots n}$ (i.e., one of the lines is $\left.\left(E_{1}, \ldots, E_{n}\right)\right)$

Case (i). Then the right hand side determinant in (20) is a polynomial of degree $n-p \geq 2$. Thus we can find a monomial in this determinant of the form $X_{\sigma(p+2)}^{I_{p+1}} \cdots X_{\sigma(n)}^{I_{n}}$ (where $\sigma$ is a substitution of $\{p+1, \ldots, n\}$ ) where each variable is independent of $X\lrcorner \Omega$. Hence in order to achieve (20) we must have $A_{I_{1} \cdots I_{n}}=0$.

Case (ii). We assume w.l.g. that $m^{I_{p+1}}=p_{1 \cdots n}$. We freeze the variables $X_{\mu}^{I_{p+1}}$ (i.e., $E_{\mu}$ ) suitably and specialize again property $(20)$ by letting free only the variables $X_{\mu}^{I_{j}}$ for $p+2 \leq j \leq n$ and $1 \leq \mu \leq n$. Two subcases occur: if $p<n-2$ then we choose $X_{\mu}^{I_{p+1}}=\delta_{\mu}^{p+1}$. Then we are reduced to a situation quite similar to the first case and we can conclude using the same argument (this time with a determinant which is a monomial of degree $n-1-p \geq 2)$.

If $p=n-2$ then $a(X)=A_{I_{1} \cdots I_{n}}\left(X_{n}^{I_{n}} X_{n-1}^{I_{n-1}}-X_{n-1}^{I_{n}} X_{n}^{I_{n-1}}\right)$. If the knowledge of $X\lrcorner \Omega$ prescribes $X_{n}^{I_{n}}$ then by the key observation $X_{n-1}^{I_{n}}$ is free and by choosing $X_{\mu}^{I_{n-1}}=\delta_{\mu}^{n}$ we obtain $a(X)=-A_{I_{1} \cdots I_{n}} X_{n-1}^{I_{n}}$. If $\left.X\right\lrcorner \Omega$ prescribes $X_{n-1}^{I_{n}}$ then $X_{n}^{I_{n}}$ is free and by choosing $X_{\mu}^{I_{n-1}}=\delta_{\mu}^{n-1}$ we obtain $a(X)=A_{I_{1} \cdots I_{n}} X_{n}^{I_{n}}$. In both cases we must have $A_{I_{1} \cdots I_{n}}=0$ in order to have $(20)$.

Conclusion. Steps 2 and 3 show that, on $\mathcal{O}_{m}^{1 \cdots n} \mathcal{M}, X \longmapsto a(X)$ is an affine function on the variables $X_{\mu, \beta_{1} \cdots \beta_{n}}$. Then by standard results in linear algebra (20) implies that, $\forall X \in \mathcal{O}_{m}^{1 \cdots n} \mathcal{M}, a(X)$ is an affine combination of the components of $X\lrcorner \Omega$. By repeating this step on each $\mathcal{O}_{m}^{\alpha_{1} \cdots \alpha_{n}} \mathcal{M}$ we deduce the conclusion.

Theorem 5.2. Assume that $\mathcal{N}=\mathcal{X} \times \mathcal{Y}, \mathcal{M}=\Lambda^{n} T^{*} \mathcal{N}$ and consider $\mathcal{M}^{d D W}$ to be the submanifold of $\Lambda^{n} T^{*} \mathcal{N}$ as defined in Paragraph 2.3.1 equipped with the multisymplectic form $\Omega^{d D W}$ which is the restriction of $\Omega$ to $\mathcal{M}^{d D W}$. Then $\mathfrak{P}^{n-1} \mathcal{M}^{d D W}$ coincides with $\mathfrak{P}_{0}^{n-1} \Lambda^{n} T^{*}(\mathcal{X} \times \mathcal{Y})_{\mid \mathcal{M}^{d D W}}$, the set of the restrictions of algebraic observable $(n-1)$-forms of $(\mathcal{M}, \Omega)$ to $\mathcal{M}^{d D W}$.

Proof. The fact that $\mathfrak{P}^{n} \mathcal{M}^{d D W}$ contains all the restrictions of algebraic observable $(n-1)$-forms of $(\mathcal{M}, \Omega)$ to $\mathcal{M}^{d D W}$ was observed in Paragraph 4.2.1. The proof of the reverse inclusion follows the same strategy as the proof of Theorem 5.1 and is left to the reader. 


\subsection{All algebraic observable $(n-1)$-forms}

We conclude this section by giving the expression of all algebraic observable $(n-1)$-forms on an open subset of $\Lambda^{n} T^{\star} \mathcal{N}$. For details see [16]. First the general expression of an infinitesimal symplectomorphism is $\Xi=\chi+\bar{\xi}$, where

$$
\begin{aligned}
\chi & :=\sum_{\beta_{1}<\cdots<\beta_{n}} \chi_{\beta_{1} \cdots \beta_{n}}(q) \frac{\partial}{\partial p_{\beta_{1} \cdots \beta_{n}}} \quad \text { and } \\
\bar{\xi} & :=\sum_{\alpha} \xi^{\alpha}(q) \frac{\partial}{\partial q^{\alpha}}-\sum_{\alpha, \beta} \frac{\partial \xi^{\alpha}}{\partial q^{\beta}}(q) \Pi_{\alpha}^{\beta},
\end{aligned}
$$

and where the coefficients $\chi_{\beta_{1} \cdots \beta_{n}}$ are so that $\left.d(\chi\lrcorner \Omega\right)=0, \xi:=\sum_{\alpha} \xi^{\alpha}(q) \frac{\partial}{\partial q^{\alpha}}$ is an arbitrary vector field on $\mathcal{N}$, and

$$
\Pi_{\alpha}^{\beta}:=\sum_{\beta_{1}<\cdots<\beta_{n}} \sum_{\mu} \delta_{\beta_{\mu}}^{\beta} p_{\beta_{1} \cdots \beta_{\mu-1} \alpha \beta_{\mu+1} \cdots \beta_{n}} \frac{\partial}{\partial p_{\beta_{1} \cdots \beta_{n}}} .
$$

As a consequence any algebraic observable $(n-1)$-form $F$ can be written as $F=Q^{\zeta}+P_{\xi}$, where

$$
\left.Q^{\zeta}=\sum_{\beta_{1}<\cdots<\beta_{n-1}} \zeta_{\beta_{1} \cdots \beta_{n-1}}(q) d q^{\beta_{1}} \wedge \cdots \wedge d q^{\beta_{n-1}} \quad \text { and } \quad P_{\xi}=\xi\right\lrcorner \theta .
$$

Then $\chi\lrcorner \Omega=-d Q^{\zeta}$ and $\left.\bar{\xi}\right\lrcorner \Omega=-d P_{\xi}$.

Lastly we let $^{8} \mathfrak{s p}_{Q} \mathcal{M}$ to be the set of infinitesimal symplectomorphisms of the form $\chi$ (with $\chi\lrcorner \Omega$ closed) and $\mathfrak{s p}_{P} \mathcal{M}$ those of the form $\bar{\xi}$ (for all vector fields $\xi \in \Gamma(\mathcal{M}, T \mathcal{M})$ ) as defined in (22). Then one can observe that $\mathfrak{s p}_{0} \mathcal{M}=\mathfrak{s p}_{P} \mathcal{M} \ltimes \mathfrak{s p}_{Q} \mathcal{M}$ (see [15]).

\section{Dynamical observable forms and functionals}

One question is left: to make sense of the Poisson bracket of two observable functionals supported on different slices. This is essential in an Einstein picture (classical analogue of the Heisenberg picture) which seems unavoidable in a completely covariant theory. One possible answer rests on the notion of dynamical observable forms (in contrast with kinematic observable functionals). To be more precise let $\Sigma$ and $\Sigma^{\prime}$ be two different slices of codimension

\footnotetext{
${ }^{8}$ Recall that $\mathfrak{s p}_{0} \mathcal{M}$ is the set of all symplectomorphisms of $(\mathcal{M}, \Omega)$ (see Definition 2.3).
} 
1 and $F$ and $G$ be two algebraic observable $(n-1)$-forms and let us try to define the Poisson bracket between $\int_{\Sigma} F$ and $\int_{\Sigma^{\prime}} G$.

One way is to express one of the two observable functionals, say $\int_{\Sigma^{\prime}} G$, as an integral over $\Sigma$. This can be achieved for all slices $\Sigma$ and $\Sigma^{\prime}$ which are cobordism equivalent, i.e., such that there exists a smooth domain $\mathcal{D}$ in $\mathcal{M}$ with $\partial \mathcal{D}=\Sigma^{\prime}-\Sigma$, and if $d G_{\mid \Gamma}=0, \forall \Gamma \in \mathcal{E}^{\mathcal{H}}$. Then indeed

$$
\int_{\Sigma \cap \Gamma} G-\int_{\Sigma^{\prime} \cap \Gamma} G=\int_{\partial \mathcal{D} \cap \Gamma} G=\int_{\mathcal{D} \cap \Gamma} d G=0,
$$

so that

$$
\int_{\Sigma^{\prime}} G=\int_{\Sigma} G \text { on } \mathcal{E}^{\mathcal{H}}
$$

Thus we are led to the following.

Definition 6.1. A dynamical observable $(n-1)$-form is an observable form $G \in \mathfrak{P}^{n-1} \mathcal{M}$ such that

$$
\{\mathcal{H}, G\}=0
$$

Indeed Corollary 3.1 implies immediately that if $G$ is a dynamical observable $(n-1)$-form then $d G_{\mid \Gamma}=0$ and hence (23) holds. As a consequence if $F$ is any observable $(n-1)$-form and $G$ is a dynamical observable $(n-1)$-form (and if one of both is an algebraic one), then we can state

$$
\left\{\int_{\Sigma} F, \int_{\Sigma^{\prime}} G\right\}:=\left\{\int_{\Sigma} F, \int_{\Sigma} G\right\}
$$

The concept of dynamical observable form is actually more or less the one used by J. Kijowski in [23], since his theory corresponds to working on the restriction of $(\mathcal{M}, \Omega)$ on the hypersurface $\mathcal{H}=0$.

Hence we are led to the question of characterizing dynamical observable $(n-1)$-forms. (We shall consider mostly algebraic observable forms.) This question was already investigated for some particular case in [23] (and discussed in [11]) and the answer was a (surprising) deception: as long as the variational problem is linear (i.e., the Lagrangian is a quadratic function of all variables) there are many observable functionals (basically all smeared integrals of fields using test functions which satisfy the Euler-Lagrange equation), but as soon as the problem is non linear the choice of dynamical observable forms is dramatically reduced and only global dynamical observable exists. For instance for a non nonlinear scalar field theory with $L(u, d u)=\frac{1}{2}\left(\partial_{t} u\right)^{2}-|\nabla u|^{2}+\frac{m^{2}}{2} u^{2}+\frac{\lambda}{3} u^{3}$, the only dynamical observable forms $G$ are those for which $\xi_{G}$ is a generator of the Poincaré group. One 
can also note that in general dynamical observable forms correspond to momentum or energy-momentum observable functionals.

Several possibilities may be considered to go around this difficulty. If the variational problem can be seen as a deformation of a linear one (i.e., of a free field theory) then it could be possible to construct a perturbation theory, leading to Feynman type expansions for classical fields. For an example of such a theory, see [13] and [12]. Another interesting direction would be to explore completely integrable systems. We present here a third alternative, which relies on symmetries and we will see on a simple example how the purpose of constructing dynamical observable forms leads naturally to gauge theories.

Example 8 (Complex scalar fields). We consider on the set of maps $\varphi: \mathbb{R}^{n} \longrightarrow \mathbb{C}$ the variational problem with Lagrangian

$$
\begin{aligned}
L_{0}(\varphi, d \varphi) & =\frac{1}{2} \eta^{\mu \nu} \frac{\partial \bar{\varphi}}{\partial x^{\mu}} \frac{\partial \varphi}{\partial x^{\nu}}+V\left(\frac{|\varphi|^{2}}{2}\right) \\
& =\frac{1}{2} \eta^{\mu \nu}\left(\frac{\partial \varphi^{1}}{\partial x^{\mu}} \frac{\partial \varphi^{1}}{\partial x^{\nu}}+\frac{\partial \varphi^{2}}{\partial x^{\mu}} \frac{\partial \varphi^{2}}{\partial x^{\nu}}\right)+V\left(\frac{|\varphi|^{2}}{2}\right)
\end{aligned}
$$

Here $\varphi=\varphi^{1}+i \varphi^{2}$. We consider the multisymplectic manifold $\mathcal{M}_{0}$, with coordinates $x^{\mu}, \phi^{1}, \phi^{2}, e, p_{1}^{\mu}$ and $p_{2}^{\mu}$ and the multisymplectic form $\Omega_{0}=$ de $\wedge \omega+d p_{a}^{\mu} \wedge d \phi^{a} \wedge \omega_{\mu}$ (which is the differential of the Poincaré-Cartan form $\left.\theta_{0}:=e \omega+p_{a}^{\mu} d \phi^{a} \wedge \omega_{\mu}\right)$. Then the Hamiltonian is

$$
\mathcal{H}_{0}(x, \phi, e, p)=e+\frac{1}{2} \eta_{\mu \nu}\left(p_{1}^{\mu} p_{1}^{\nu}+p_{2}^{\mu} p_{2}^{\nu}\right)-V\left(\frac{|\phi|^{2}}{2}\right) .
$$

We look for $(n-1)$-forms $F_{0}$ on $\mathcal{M}_{0}$ such that

$$
\begin{gathered}
\left.d F_{0}+\xi_{F_{0}}\right\lrcorner \Omega_{0}=0, \quad \text { for some vector field } \xi_{F_{0}}, \\
d \mathcal{H}_{0}\left(\xi_{F_{0}}\right)=0 .
\end{gathered}
$$

The analysis of this problem can be dealt by looking for all vector fields

$$
\begin{aligned}
\xi_{0}=X^{\mu}(x, \phi, e, p) \frac{\partial}{\partial x^{\mu}}+\Phi^{a}(x, \phi, & , p) \frac{\partial}{\partial \phi^{a}} \\
& +E(x, \phi, e, p) \frac{\partial}{\partial e}+P_{a}^{\mu}(x, \phi, e, p) \frac{\partial}{\partial p_{a}^{\mu}} .
\end{aligned}
$$

satisfying (24) and (25). For simplicity we will assume that $X^{\mu}=0$ (this will exclude stress-energy tensor observable forms $X^{\mu} \frac{\partial}{\partial x^{\mu}}-\theta_{0}$, for $X^{\mu}$ constant). Then we find two cases: 
If $V\left(|\phi|^{2} / 2\right)$ is quadratic in $\phi$, i.e., if $V\left(|\phi|^{2} / 2\right)=m^{2}|\phi|^{2} / 2$, then Equations (24) and (24) have the solutions

$\xi_{0}=\lambda \vec{j}_{0}+U^{a}(x) \frac{\partial}{\partial \phi^{a}}-\left(p_{a}^{\mu} \frac{\partial U^{a}}{\partial x^{\mu}}(x)+\delta_{a b} L U^{a}(x) \phi^{b}\right) \frac{\partial}{\partial e}+\eta^{\mu \nu} \delta_{a b} \frac{\partial U^{a}}{\partial x^{\mu}}(x) \frac{\partial}{\partial p_{b}^{\mu}}$,

where $\lambda$ is a real constant,

$$
\vec{j}_{0}:=\left(\phi^{2} \frac{\partial}{\partial \phi^{1}}-\phi^{1} \frac{\partial}{\partial \phi^{2}}\right)+\left(p_{2}^{\mu} \frac{\partial}{\partial p_{1}^{\mu}}-p_{1}^{\mu} \frac{\partial}{\partial p_{2}^{\mu}}\right)
$$

$L:=-\eta^{\mu \nu} \frac{\partial^{2}}{\partial x^{\mu} \partial x^{\nu}}$ and $U^{1}$ and $U^{2}$ are arbitrary solutions of the linear equation $L U+m^{2} U=0$. Then $F_{0}=U^{a} p_{a}^{\mu} \omega_{\mu}-\eta^{\mu \nu}\left(\frac{\partial U^{1}}{\partial x^{\nu}} \phi^{1}+\frac{\partial U^{2}}{\partial x^{\nu}} \phi^{2}\right) \omega_{\mu}+$ $\lambda\left(p_{1}^{\mu} \phi^{2}-p_{2}^{\mu} \phi^{1}\right) \omega_{\mu}$.

However if $V^{\prime}$ is not a constant, then system (24) and (24) has only the solutions $\xi_{0}=\lambda \vec{j}_{0}$ and the resulting dynamical observable $(n-1)$-form is $F_{0}=\lambda\left(p_{1}^{\mu} \phi^{2}-p_{2}^{\mu} \phi^{1}\right) \omega_{\mu}$, which corresponds to the global charge due to the $U(1)$ invariance of the Lagrangian.

For instance we would like to replace $\lambda$ by a smooth function $\psi$ of $x$, i.e., to look at $F=\psi(x)\left(p_{1}^{\mu} \phi^{2}-p_{2}^{\mu} \phi^{1}\right) \omega_{\mu}$. These are non dynamical algebraic observable $(n-1)$-forms since we have $\left.d F_{1}+\widetilde{\xi}\right\lrcorner \Omega_{0}=0$, where $\widetilde{\xi}:=\psi \overrightarrow{j_{0}}-$ $\left(p_{1}^{\mu} \phi^{2}-p_{2}^{\mu} \phi^{1}\right) \frac{\partial \psi}{\partial x^{\mu}} \frac{\partial}{\partial e}$, but $d \mathcal{H}_{0}(\widetilde{\xi})=-\left(p_{1}^{\mu} \phi^{2}-p_{2}^{\mu} \phi^{2}\right) \frac{\partial \psi}{\partial x^{\mu}} \neq 0$.

Now in order to enlarge the set of dynamical observable forms, an idea is to further incorporate the gauge potential field $A:=A_{\mu} d x^{\mu}$ and consider the Lagrangian

$$
\begin{aligned}
L_{1}(\varphi, A, d \varphi):= & \frac{1}{2} \eta^{\mu \nu}\left(\overline{\frac{\partial \varphi}{\partial x^{\mu}}+i A_{\mu} \varphi}\right)\left(\frac{\partial \varphi}{\partial x^{\nu}}+i A_{\nu} \varphi\right) \\
& -\frac{1}{4} \eta^{\mu \lambda} \eta^{\nu \sigma} F_{\mu \nu} F_{\lambda \sigma}+V\left(\frac{|\varphi|^{2}}{2}\right),
\end{aligned}
$$

where $F_{\mu \nu}:=\frac{\partial A_{\nu}}{\partial x^{\mu}}-\frac{\partial A_{\mu}}{\partial x^{\nu}}$. It is invariant under gauge transformations $\varphi \longmapsto e^{i \theta} \varphi, A \stackrel{\partial}{\longmapsto} A-d \theta$. Note that we did incorporate an energy for the gauge potential $A$. We now consider the multisymplectic manifold $\mathcal{M}_{1}$ with coordinates $x^{\mu}, \phi^{1}, \phi^{2}, e, p_{1}^{\mu}, p_{2}^{\mu}, a_{\mu}$ and $p^{\mu \nu}$. The multisymplectic form is: $\Omega_{1}=d e \wedge \omega+d p_{a}^{\mu} \wedge d \phi^{a} \wedge \omega_{\mu}-\left(d a_{\lambda} \wedge d x^{\lambda}\right) \wedge\left(\frac{1}{2} d p^{\mu \nu} \wedge \omega_{\mu \nu}\right)$. The Hamiltonian is then

$$
\begin{aligned}
\mathcal{H}_{1}(x, \phi, a, e, p)= & e+\frac{1}{2} \eta_{\mu \nu}\left(p_{1}^{\mu} p_{1}^{\nu}+p_{2}^{\mu} p_{2}^{\nu}\right)+\left(p_{1}^{\mu} \phi^{2}-p_{2}^{\mu} \phi^{1}\right) a_{\mu} \\
& -\frac{1}{4} \eta_{\mu \lambda} \eta_{\nu \sigma} p^{\mu \nu} p^{\lambda \sigma}-V\left(\frac{|\phi|^{2}}{2}\right) .
\end{aligned}
$$


The gain is that we may now consider the algebraic observable $(n-1)$-form

$$
F_{1}:=\psi(x)\left(p_{1}^{\mu} \phi^{2}-p_{2}^{\mu} \phi^{1}\right) \omega_{\mu}-\frac{1}{2} p^{\mu \nu} d \psi \wedge \omega_{\mu \nu} .
$$

where $\psi$ is any smooth function of $x$. We indeed still have on the one hand $\left.d F_{1}=-\xi_{1}\right\lrcorner \Omega_{1}$, where

$$
\xi_{1}:=\psi \vec{j}_{0}-\left(p_{1}^{\mu} \phi^{2}-p_{2}^{\mu} \phi^{1}\right) \frac{\partial \psi}{\partial x^{\mu}} \frac{\partial}{\partial e}+\frac{\partial \psi}{\partial x^{\mu}} \frac{\partial}{\partial a_{\mu}} .
$$

Then $d \mathcal{H}_{1}\left(\xi_{F_{1}}\right)=0$. Thus $F_{1}$ is a dynamical observable $(n-1)$-form.

\section{References}

[1] E. Binz, J. Śnyatycki and H. Fisher, The geometry of classical fields, North Holland, Amsterdam, 1989.

[2] R.L. Bryant, S.S. Chern, R.B. Gardner, H.L. Goldschmidt and P.A. Griffiths, Exterior differential systems, Springer-Verlag, MSRI, 18, 1991.

[3] C. Carathéodory, Variationsrechnung und partielle Differentialgleichungen erster Ordnung, Teubner, Leipzig (reprinted by Chelsea, New York, 1982).

[4] M. Castrillon opez and J.E. Marsden, Some remarks on Lagrangian and Poisson reduction for field Theories, J. Geom. Phys. 48 (2003), 52-83.

[5] T. de Donder, Théorie invariante du calcul des variations, GauthiersVillars, Paris, 1930.

[6] P. Dedecker, Calcul des variations, formes différentielles et champs géodésiques, in 'Géométrie différentielle', Colloq. Intern. du CNRS LII, Strasbourg 1953, Publ. du CNRS, Paris, 1953, 17-34;

On the generalization of symplectic geometry to multiple integrals in the calculus of variations, in 'Differential Geometrical Methods in Mathematical Physics', eds. K. Bleuler and A. Reetz, Lect. Notes Maths., 570, Springer-Verlag, Berlin, 1977, 395-456.

[7] M. Forger and H. Römer, A Poisson bracket on multisymplectic phase space, Rep. Math. Phys. 48 (2001), 211-218; arXiv:math-ph/0009037.

[8] M. Forger, C. Paufler and H. Römer, The Poisson bracket for Poisson forms in multisymplectic theories, arXiv:math-ph/0202043. 
[9] K. Gawędski, On the generalization of the canonical formalism in the classical field theory, Rep. Math. Phys. 4(3) (1972), 307-326.

[10] M.J. Gotay, J. Isenberg and J.E. Marsden (with the collaboraton of R. Montgomery, J. Śnyatycki, P.B. Yasskin), Momentum maps and classical relativistic fields, Part I: covariant field theory, preprint, arXiv:physics/9801019.

[11] H. Goldschmidt and S. Sternberg, The Hamilton-Cartan formalism in the calculus of variations, Ann. Inst. Fourier Grenoble 23(1) (1973), 203-267.

[12] R. Harrivel, Planar binary trees and pertubative calculus of observables in classical field theory, preprint, arXiv:math.AP/0410050.

[13] F. Hélein, Hamiltonian formalisms for multidimensional calculus of variations and perturbation theory, Contemporary Math. 350 (2004), $127-147$.

[14] F. Hélein and J. Kouneiher, Finite dimensional Hamiltonian formalism for gauge and quantum field theory, J. Math. Physics 43(5) (2002).

[15] F. Hélein and J. Kouneiher, Covariant Hamiltonian formalisms for the calculus of variations with several variables, extended version, arXiv:math-ph/0211046.

[16] F. Hélein and J. Kouneiher, Covariant Hamiltonian formalisms for the calculus of variations with several variables: Lepage-Dedecker versus de Donder-Weyl, Adv. Theor. Math. Phys. 8 (2004), 575-611.

[17] F. Hélein and J. Kouneiher, Covariant multisymplectic formulations of gauge theories, in preparation.

[18] F. Hélein and J. Kouneiher, Multisymplectic versus pataplectic manifolds, in preparation.

[19] S. Hrabak, On a multisymplectic formulation of the classical BRST symmetry for first order field theories, Part I: Algebraic structure, arXiv:math-ph/9901012.

[20] S. Hrabak, On a multisymplectic formulation of the classical BRST symmetry for first order field theories, Part II: Geometric structure, arXiv:math-ph/9901013.

[21] I.V. Kanatchikov, Canonical structure of classical field theory in the polymomentum phase space, Rep. Math. Phys. 41(1) (1998); arXiv:hepth/9709229. 
[22] I.V. Kanatchikov, On field theoretic generalizations of a Poisson algebra, preprint, arXiv:hep-th/9710069.

[23] J. Kijowski, A finite dimensional canonical formalism in the classical field theory, Comm. Math. Phys. 30 (1973), 99-128.

[24] J. Kijowski and W. Szczyrba, A canonical structure for classical field theories, Comm. Math. Phys. 46 (1976), 183-206.

[25] J. Kijowski and W.M. Tulczyjew, A symplectic framework for field theories, Springer-Verlag, Berlin, 1979.

[26] C. Paufler, A vertical exterior derivative in multisymplectic fields and the generalized Poisson bracket for non trivial geometries, to appear in J. Geom. Phys.; arXiv:math-ph/0002032.

[27] C. Rovelli, A note on the foundation of relativistic mechanics - II: Covariant Hamiltonian general relativity, arXiv:gr-qc/0202079.

[28] W.M. Tulczyjew, Geometry of phase space, seminar in Warsaw, 1968, unpublished.

[29] W.M. Tulczyjew, The graded Lie algebra of multivector fields and the generalized Lie derivative of forms, Bull. de l'Acad. Polon. des Sci., Série sci. Math., Astr. et Phys. XXII (1974), 937-942.

[30] H. Weyl, Geodesic fields in the calculus of variation for multiple integrals, Ann. Math. 6 (1935), 607-629. 